\title{
Erwerbstätige mit privaten Pflegeaufgaben am Beispiel zweier schweizerischer Unternehmen
}

\author{
Andrea Radvanszky*, Julie Craviolini** und Iren Bischofberger* \\ * Careum Forschung, Kalaidos Fachhochschule Gesundheit, CH-8032 Zürich, andrea.radvanszky@ \\ careum.ch und iren.bischofberger@careum.ch. \\ ** Statistisches Amt des Kantons Zürich, CH-8090 Zürich, julie.craviolini@statistik.ji.zh.ch.
}

\begin{abstract}
s
Erwerbstätige mit privaten Pflegeaufgaben am Beispiel zweier schweizerischer Unternehmen

In Zukunft werden mehr Personen mit privaten Pflege- und Betreuungsaufgaben im Erwerbsprozess stehen. Über diese Gruppe ist in der Schweiz bislang wenig bekannt. Was das Vereinbarkeitsphänomen und das Zusammenwirken der einschlägigen Indikatoren betrifft, besteht ein erheblicher Erkenntnisund Forschungsbedarf. In diesem Beitrag werden verschiedene Faktoren für die Pflegeübernahme und den Pflegeumfang mittels zweier Erhebungen in einer öffentlichen Verwaltung und einem privaten Unternehmen multivariat untersucht. Für die Pflegeübernahme erweisen sich das Alter, das Geschlecht und die Lohnhöhe als relevant, während der Pflegeumfang mit Charakteristika, die die Pflegesituation betreffen, korreliert. Gleichzeitig verdeutlichen die Divergenzen zwischen den Unternehmen den Effekt struktureller Variablen, die eine differenzierte Betrachtung vereinbarkeitsfördernder Massnahmen erlauben.

Schlüsselwörter: Erwerbstätigkeit, Pflege, betriebliche Angebote, Unternehmensbefragung, multivariate Analyse

\section{Soigner un proche et travailler : I'exemple de deux entreprises suisses}

Le nombre de personnes actives ayant à leur charge un proche nécessitant un accompagnement et des soins augmentera dans le futur. Les connaissances sur ce groupe sont encore insuffisantes en Suisse, notamment en ce qui concerne le phénomène de conciliation et l'interaction des indicateurs pertinents. Sur la base de données empiriques récoltées auprès d'une administration publique et d'une entreprise privée, nous considérons par une analyse multivariée les différents facteurs influençant la prise en charge des soins et l'étendue des soins apportés. L'âge, le sexe et le niveau de salaire sont déterminants dans la prise en charge des soins, tandis que l'étendue des soins apportés est particulièrement corrélée aux caractéristiques de la situation de soin. En outre, les différences entre les deux entreprises illustrent l'effet des variables structurelles permettant une vue différenciée sur les mesures en faveur d'une meilleure conciliation.
\end{abstract}

Mots-clés: Travail, soins, mesures proposées par l'entreprise, enquête dans une entreprise, analyse multivariée

\section{Employees with Caregiving Responsibilities: The Example of Two Employers in Switzerland}

In the future, more persons in the workforce will provide care and assistance to a family member while being employed. Little is known about this group in Switzerland. Comprehensive research has to be conducted on the phenomenon of the reconciliation and the interrelation of pertinent indicators. This paper describes a multivariate analysis of factors influencing the commitment and extent of family care, based on two surveys among employees in a public administration and a private company. Age, gender and wage level are relevant factors for the commitment to care, while characteristics of specific care situations correlate with the provided extent of care. Also, divergences between the two employers illustrate the effect of structural variables, allowing a closer insight into measures supporting reconciliation between work and care.

Keywords: Employment, care, corporate services, company survey, multivariate analysis 


\title{
Erwerbstätige mit privaten Pflegeaufgaben am Beispiel zweier schweizerischer Unternehmen ${ }^{1}$
}

\author{
Andrea Radvanszky*, Julie Craviolini* ${ }^{*}$ und Iren Bischofberger*
}

\section{$1 \quad$ Einleitung}

Die privat geleistete Pflege- und Betreuungsarbeit gewinnt besonders wegen der demografischen Alterung an Aufmerksamkeit. In Zukunft wird mit einer steigenden Anzahl an pflegebedürftigen Personen gerechnet (European Commission 2013; BFS 2013a). ${ }^{2}$ Bei der häuslichen aber auch stationären Versorgung von pflege- oder hilfebedürftigen Personen spielen vor allem die engsten Familienmitglieder eine tragende Rolle (Hoff und Hamblin 2011; Perrig-Chiello 2012a; Baumbusch und Phinney 2014). Als wesentliche konstitutive Elemente der familiären Hilfebeziehung gelten die wertrationale Fürsorgepflicht (Dallinger 1997) und die emotionale Bindung (Mnich et al. 2008). Diese Motive vermögen mit zu erklären, weshalb heute in der Schweiz viele ältere Pflegebedürftige, aber auch jüngere Personen mit dauerhaften Gesundheitsbeeinträchtigungen im Privathaushalt versorgt werden (BFS 2011; BFS 2012). Alterspflege in der Schweiz wird aber im Vergleich zu den Nachbarländern häufiger stationär erbracht oder mit Beteiligung ambulanter professioneller Hilfe. Der Anteil älterer Personen die durch die Kindergeneration betreut wird, ist darum deutlich geringer als etwa in Deutschland (Perrig-Chiello 2012b; Destatis 2013). Wegen der wachsenden Anzahl von Hochaltrigen werden in Zukunft relativ mehr Frauen und Männer der mittleren Generation (50- bis 64-Jährige) mit einer familialen Pflegesituation bzw. der Elternpflege herausgefordert sein. Diese Verknappung der familialen Hilfe- und Pflegeressourcen dürfte dazu führen, dass sich der Anteil

\footnotetext{
* Careum Forschung, Kalaidos Fachhochschule Gesundheit, CH-8032 Zürich, andrea.radvanszky@ careum.ch und iren.bischofberger@careum.ch.

** Statistisches Amt des Kantons Zürich, CH-8090 Zürich, julie.craviolini@statistik.ji.zh.ch.

1 Wir danken Ulrich Otto, Marc Höglinger und den anonymen GutachterInnen für inhaltliche Anmerkungen. Bei Jacqueline Schön-Bühlmann bedanken wir uns für die bereitwillige Auskunft bei der Identifizierung und Quantifizierung der pflegenden Erwerbstätigen in der Schweiz.

2 Trotz Zunahme der Anzahl von Hochaltrigen (80-jährige und ältere Personen) muss der Anteil an Pflegebedürftigen wegen des länger dauernden relativ guten Gesundheitszustands der älteren Bevölkerungsgruppe und der längeren Lebenserwartung nicht zwingend zunehmen (BFS 2013b). Auch gibt es keinen Hinweis darauf, dass sich die zu erwartende Pflege- und Betreuungsdauer verlängert hat. Dennoch ist davon auszugehen, dass auch viele Personen der jüngeren Altersgruppe (60- bis 79-Jährige) eine gelegentliche Unterstützung benötigen, die nicht allein durch das Gesundheitssystem abgedeckt wird (Schweizerische Eidgenossenschaft 2014).
} 
der formellen Versorgungsleistungen gegenüber der familialen Hilfe weiter erhöhen wird (Höpflinger 2012).

Vor diesem demografischen Hintergrund wie auch mit besonderem Blick auf die Erhöhung der Erwerbstätigkeit der Frauen, aber auch die generell steigende Erwerbsbeteiligung künftiger Alterskohorten wird ein steigender Anteil an Erwerbstätigen erwartet, die gleichzeitig die Sorge für pflege- und hilfsbedürftige Angehörige übernehmen (Schneekloth und Wahl 2005; Barkholdt und Lasch 2006; Höpflinger 2012). Von einer solchen Vereinbarkeitssituation sind insbesondere Frauen und die ältere Erwerbsbevölkerung betroffen (European Commission 2013). Pflegende Erwerbstätige stehen aufgrund von Zeit- und Rollenkonflikten in einem besonderen Spannungsfeld mit negativen Auswirkungen auf die Arbeitsleistung oder die Erwerbstätigkeit, deren Unterbrechung oder vollständige Aufgabe droht (Scharlach 1994; Creedon 1995; Arksey 2002; Martin-Matthews und Phillips 2008; Keck 2012). Auf der nationalen und internationalen politischen Ebene wird ein deutlicher Handlungsbedarf zur Verbesserung der Situation von Erwerbstätigen mit Pflegeverantwortung konstatiert (Schweizerische Eidgenossenschaft 2014; Deutscher Bundestag 2014; Department of Health 2014).

Für die Schweiz wird die Anzahl der Erwerbstätigen mit privaten Pflegeaufgaben insgesamt auf rund 445000 Personen beziehungsweise knapp 11\% der erwerbsfähigen Bevölkerung geschätzt. Davon leben etwa 88200 Personen mit einem pflegebedürftigen Haushaltsmitglied zusammen, während rund 58300 Personen Pflegeaufgaben für Verwandte und Bekannte leisten, die nicht im eigenen Haushalt leben. Rund 311000 Personen übernehmen Dienstleistungen wie Hausarbeiten, Transportdienste usw. für Verwandte und Bekannte ausserhalb des eigenen Haushalts (BFS 2010). ${ }^{3}$ Obwohl auch hierzulande diese neue und im Vergleich zur Kindererziehung anders gelagerte Form der Vereinbarkeit bislang wenig etabliert und konzeptualisiert ist, rückt sie gesellschaftspolitisch und auch in der Arbeitswelt zunehmend stärker ins Blickfeld (Bischofberger 2012).

\subsection{Lücken in der Vereinbarkeitsforschung und das Ziel der Untersuchung}

Was die Entscheidung, Pflege zu übernehmen und die Entscheidung zur Erwerbspartizipation betrifft, werden gemeinhin verschiedene soziodemografische, pflege- und erwerbsspezifische Merkmale untersucht (Franke und Reichert 2011). Die Frage, ob überhaupt, zu welchem Zeitpunkt oder wodurch konkret die Erwerbstätigkeit einer Person mit Pflegeverantwortung negativ tangiert wird, erweist sich indes ungleich schwieriger zu beantworten.

Dies hat erstens mit fehlenden Längsschnittanalysen zu tun. So zeigt Heitmueller (2007) auf, dass der in Querschnittuntersuchungen gemessene exogene Effekt der Pflegeübernahme auf die Erwerbspartizipation deutlich überschätzt wird, während andere endogene Determinanten unberücksichtigt bleiben, etwa fehlende

3 Die Angaben beruhen auf Schätzungen, weshalb die addierten Anteile von der Summe abweichen. 
Erwerbsmöglichkeiten oder Arbeitsmarktchancen. Früher getroffene Erwerbsentscheidungen und Entscheidungen für die Pflegetätigkeit können sich folglich auf ein im Lebenslauf später getroffenes Vereinbarkeitsarrangement auswirken. Obwohl in der Vereinbarkeitsdiskussion die negativen Konsequenzen der Pflege auf die Erwerbstätigkeit im Brennpunkt stehen, ist gemäss Heitmueller bei der Erwerbstätigkeit und Pflege nicht von einem Kausalitätsverhältnis auszugehen.

Zweitens erschweren neben diesem methodischen Problem terminologische Divergenzen von «Pflege» und «Erwerbstätigkeit» eine genaue Analyse der Faktoren auf die Vereinbarkeit. Dies betrifft hauptsächlich die Frage, ob Personen, die gleichzeitig pflegen und erwerbstätig sind, aus der Gruppe der Erwerbstätigen oder derjenigen der Pflegenden rekrutiert werden, aber auch, ob die Studien ausschliesslich Frauen oder Pflegekonstellationen mit älteren Pflegebedürftigen (meist über 60 Jahre alt) berücksichtigen.

Drittens ist «Vereinbarkeit», angelehnt an die wegweisende Untersuchung von Dallinger (1997), als ein Aushandlungs- und Entscheidungsprozess bei der Bewältigung des Beruf-Pflege-Konflikts zu verstehen. Wie oder woran sich die Akteure in diesem spezifischen Spannungsfeld zwischen Erwerbstätigkeit und Pflegeaufgaben orientieren, darüber geben die soziodemografischen, pflege- und erwerbsspezifischen Merkmale keinen Aufschluss. Darum teilen wir die Aussage von Franke und Reichert (2011) nicht, wonach eine Vereinbarkeitssituation mit den typischen Merkmalen "geringer Grad an Hilfebedürftigkeit, vorhandenes Hilfenetz, günstige ökonomische Lage und höheres berufliches Qualifikationsniveau der erwerbstätigen pflegenden Person und die Absenz minderjähriger Kinder» als erfolgreich und konfliktfrei gilt. Dies aus folgenden Gründen:

, Einem so verstandenen Konzept der Vereinbarkeit liegt ein normatives Verständnis zugrunde, das einen möglichen Idealzustand suggeriert.

, Neben den soziodemografischen, pflege- und erwerbsspezifischen Merkmalen begründen individuelle Präferenzen, Interessen und Bewertungen die Entscheidung, wie die Vereinbarkeitsproblematik gelöst wird.

, Pflegesituationen im Kontext chronischer Krankheiten zeichnen sich durch Unabsehbarkeit und Unplanbarkeit aus (Corbin et al. 2009), weshalb nicht von einem stabilen Arrangement ausgegangen werden kann. Inwieweit und wie lange sich ein bestimmtes Arrangement durchhalten lässt, ist mit der Übernahme von Pflegeaufgaben nicht entschieden. Zwischen Erwerbstätigkeit und Pflege muss immer wieder neu abgewogen werden.

In der Schweiz besteht, anders als in anderen europäischen Ländern (European Commission 2013) oder in den USA (NAC AARP 2009) bei der Quantifizierung von pflegenden Erwerbstätigen eine erhebliche Forschungslücke. Personen, die aufgrund ihrer Pflege- und Betreuungstätigkeit die Erwerbstätigkeit aufgegeben haben, werden nicht erfasst. Es fehlen populationsbezogene Daten, um die Situation der 
pflegenden Erwerbstätigen unter Miteinbezug der genannten Indikatoren auszuleuchten. Diese Lücke ansatzweise zu füllen, ist das Ziel unseres Aufsatzes. Auf der Grundlage von Daten aus zwei schweizerischen Unternehmen, deren Mitarbeitende im Jahr 2011 mittels einer Online-Erhebung einmalig befragt wurden, wollen wir die Gruppe der Beschäftigten mit privaten Pflege- und Betreuungsverpflichtungen im Vergleich zu jener ohne pflegerische Aufgaben anhand individueller, anstellungs- und pflegespezifischer Faktoren explorativ untersuchen. Diese zur Diskussion stehenden Faktoren verstehen wir aus den genannten Gründen nicht als «Vereinbarkeitsindikatoren», d.h., wir treffen keine Aussagen zum Zusammenhang von Pflege- und Erwerbsentscheidungen. Stattdessen fragen wir danach, welche Faktoren auf die Übernahmewahrscheinlichkeit von Pflegeaufgaben und den Pflegeumfang einwirken. Neben dem Profil der pflegenden Erwerbstätigen interessiert uns der Pflegeumfang, weil sich dieser als ein zentraler Faktor für die Erwerbspartizipation erweist (Carmichael und Charles 1998; Carmichael et al. 2010). Wir gehen davon aus, dass sich Erwerbstätige mit privaten Pflegeaufgaben materiell und zeitlich engagieren und folgen somit dem ökonomischen Ansatz von Heitmueller (2007), Carmichael et al. (2010) und Schneider et al. (2001). ${ }^{4}$ Pflege- und Betreuungsaufgaben zu übernehmen bedeutet einerseits, finanziell Einbussen in Kauf zu nehmen, andererseits stehen diese Aufgaben in einem zeitlichen Konkurrenzverhältnis zur Erwerbstätigkeit und der eigenen Freizeit.

Schliesslich wollen wir mittels der beiden Unternehmensstichproben untersuchen, inwieweit die Betriebsstruktur die Unterschiede bei der Pflegeübernahme und beim Pflegeumfang der Mitarbeitenden zu erklären vermag. Weil betriebliche Fördermassnahmen wie flexible Arbeitszeiten oder Abwesenheitsregelungen als Hauptfaktoren gelten, um Pflege und Erwerbstätigkeit miteinander zu vereinbaren (European Commission 2013), interessiert uns überdies, welche Vereinbarkeitsangebote pflegende Mitarbeitende in Anspruch nehmen. Diese Angebote stellen wir den unterschiedlichen Betriebsstrukturen entgegen, wobei wir davon ausgehen, dass sie je nach Betriebsstruktur unterschiedlich greifen.

$4 \quad$ Für diese theoretische Verortung sprechen auch die Befunde der wissenssoziologisch orientierten, qualitativen Studie Dallingers (1997), wonach bei der Pflegeübernahme Erwägungen zu Opportunitätskosten und zur Sicherung des familiären ökonomischen Kapitals eine wichtige Rolle spielen. Zudem wird "zeitlich» abgewogen, beispielsweise zwischen der eigenen Lebenszeit und der Lebenszeit der pflegebedürftigen Person und zwischen Pflegeaufgaben und Erwerbstätigkeit, während Teilzeitarbeit als eine die Nachteile des Pflegeengagements begrenzende Handlungsstrategie gewählt wird. 


\section{Einflussfaktoren auf die Pflegeübernahme und den Pflegeumfang}

\subsection{Merkmale der erwerbstätigen Person, des Beschäftigungsverhältnisses und der Pflegesituation}

Untersuchungen zum Effekt des Pflegeengagements auf die Erwerbspartizipation beruhen auf der Basisannahme des zeitlichen Konkurrenzverhältnisses. Ökonomisch betrachtet führt die Pflegesituation zu knapperen Zeitressourcen für andere Lebensbereiche wie Erwerbsarbeit und Freizeit. Es kommt zu einer Abwägung und Neuordnung von Präferenzen. Bei der Frage, ob Pflege übernommen wird oder nicht, spielen allerdings bestimmte Bedingungen eine Rolle, die den individuellen Entscheidungsspielraum erheblich einschränken können, beispielsweise eine Pflegesituation im gleichen Haushalt, wo es schwierig ist, sich den nötigen Handreichungen zu entziehen.

Längsschnittuntersuchungen für England und Deutschland kommen zum gleichen Schluss: Pflegesituationen im gleichen Haushalt und ein hoher Pflegeumfang - festgelegt auf über 20 Stunden pro Woche - wirken sich negativ auf die Erwerbspartizipation aus (Schneider et al. 2001; Heitmueller 2007; Carmichael et al. 2010). Dass es besonders in diesen Fällen schwierig ist, Pflege mit Erwerbstätigkeit zu kombinieren, verdeutlicht das Problem der Zeitknappheit. Bei Pflegesituationen im gleichen Haushalt und mit hohem Pflegeaufwand besteht gemäss diesen Studien kaum Entscheidungsspielraum; trotz eigener Einkommenseinbussen wird Pflege übernommen.

Dennoch zeigen sich nicht nur erwerbstätige Personen an sich, sondern auch Personen mit einem höheren Stundenlohn weniger bereit, Pflegeaufgaben zu übernehmen. Die Opportunitätskosten der Pflege sind für Personen mit hohem Einkommen entsprechend hoch (Schneider et al. 2001; Carmichael et al. 2010). Die Übernahmeentscheidung impliziert insofern auch ein rationales Element. Der Entscheidungsspielraum der Pflegeübernahme wird zudem durch das Verwandtschaftsverhältnis eingeschränkt. Im Vergleich zur (Schwieger-)Elternpflege oder der Pflege von Nachbarn oder Freunden konstatiert Heitmueller (2007) bei der Partnerpflege den negativsten Effekt auf die Erwerbstätigkeit. ${ }^{5}$

Bei der Pflegeübernahme wirken auch endogene Effekte. Personen mit eingeschränkten Arbeitsmarktchancen, Diskontinuitäten in der Erwerbstätigkeit oder mit Pflegeerfahrung neigen eher dazu, Pflegeaufgaben zu übernehmen (Heitmueller 2007). Mit dieser prozessualen Perspektive lassen sich auch die Geschlechterunter-

$5 \quad$ Heitmueller (2007) untersucht Pflegefälle im gleichen Haushalt und haushaltsübergreifend. Erstere beziehen sich v. a. auf Partnerpflege. Bei Schneider et al. (2001) aber sind haushaltsübergreifende Pflegeleistungen ausgeklammert und es dominieren intergenerationelle Pflegekonstellationen (Eltern-/Schwiegerelternpflege). Wie und ob der exogene Einfluss durch das Zusammenspiel der Variablen Pflegesituation innerhalb / ausserhalb des Haushalts und Verwandtschaftsgrad weiter verstärkt wirkt, ist unklar. Offensichtlich handelt es sich aber um unterschiedliche Faktoren, die auf die Pflegeentscheidung einwirken. 
schiede in der Pflege exakter fassen, weil die rollentheoretische Erklärung um ein haushaltsökonomisches Argument erweitert wird. Schneider et al. (2001) weisen nach, dass mit steigendem Haushaltseinkommen voll- wie auch teilzeiterwerbstätige verheiratete Frauen eine erhöhte Neigung aufweisen, aus dem Erwerbsprozess auszusteigen, wenn eine Pflegesituation im Haushalt eintritt. Die Entscheidung, die Erwerbsarbeit zugunsten der Pflegeübernahme zu unterbrechen, hat deshalb massgeblich mit der finanziellen Absicherung durch den Ehepartner zu tun. Zwar geben in Teilzeit erwerbstätige Frauen mit hohem persönlichem Einkommen ihre Erwerbstätigkeit weniger auf - Opportunitätskosten spielen auch hier eine Rolle. Je höher aber das Haushaltseinkommen ist, umso eher wird die Teilzeit-Erwerbstätigkeit unterbrochen. Bei den in Vollzeit erwerbstätigen Frauen wirken sich die Anzahl Jahre in Vollzeitbeschäftigung und die Einkommenshöhe negativ auf die Aufgabe der Erwerbstätigkeit aus. Je höher aber das Haushaltseinkommen ist, umso eher wird das Erwerbspensum reduziert oder ganz aufgegeben. Für unsere Untersuchung können wir einen Effekt des Haushaltseinkommens auf die Pflegeübernahme annehmen. Weil aber Schneider et al. (2001) nur die Population der verheirateten Frauen betrachten, ist die Richtung des Effekts unklar.

Die Befunde beim Bildungsniveau sind nicht eindeutig, was Schneider et al. (2001) damit erklären, dass die Variable einerseits auf den Humankapitaleffekt und andererseits auf den Homogamieeffekt verweisen kann. Besser Ausgebildete haben aufgrund ihres höheren Einkommens mehr Versorgungsalternativen, weshalb sie selber weniger (oder möglicherweise weniger zeitintensive) Pflegetätigkeit übernehmen. Aber auch höher Gebildete unterbrechen aufgrund des geringeren ökonomischen Drucks ihre Erwerbstätigkeit wegen Pflegeaufgaben, was insbesondere auf Frauen zutrifft. Hieran zeigt sich die Problematik, wenn aus dem Erwerbsprozess Ausgeschiedene in den Daten nicht repräsentiert sind.

Gemäss Carmichael et al. (2010) zeigt das Erwerbspensum der Frauen nur einen schwachen Effekt auf die Übernahmewahrscheinlichkeit im Falle von hohem Pflegeumfang und Pflegesituationen im gleichen Haushalt. Weibliche Teilzeit Angestellte übernehmen im Vergleich zu weiblichen Vollzeit Angestellten leicht häufiger Pflegeaufgaben. Die Schlussfolgerung, dass Frauen pflegen, weil sie in Teilzeit arbeiten, ist aber so nicht zulässig. Mit Heitmueller (2007) gesprochen, führt die Diskontinuität in der Erwerbstätigkeit bei den Frauen, also die unterschiedliche Erwerbspositionierung von Frauen und Männern dazu, dass Frauen (auch im Falle hoher Opportunitätskosten) ihre Erwerbstätigkeit zugunsten der Pflege aufgeben bzw. anpassen. Inwieweit dieser wichtige Befund zu verallgemeinern bzw. auf Pflegekonstellationen zu übertragen ist, die haushaltsübergreifend stattfinden, bleibt aber offen.

Erwerbsentscheidungen, die durch eine auftretende Pflegesituation getroffen werden müssen, sind demnach als ein Zusammenspiel von erwerbsbiografischen Faktoren, persönlichem Einkommen und finanziellem Hintergrund zu betrachten. Während sich ein hohes persönliches Einkommen negativ auf die Pflegeübernahme 
auswirkt, kann ein hohes Haushaltseinkommen die Pflegeübernahme im gleichen Haushalt bzw. bei hohem Pflegeaufwand begünstigen. Neben dem Wohnort der pflegebedürftigen Person erweist sich der Umfang der geleisteten Pflegestunden als eine wichtige Determinante, um Auswirkungen auf die Erwerbstätigkeit zu messen.

Die pflegespezifischen Faktoren können als «Nachfrage» nach Pflegeleistungen verstanden werden und somit einen Effekt auf den Pflegeumfang haben. Die Art der Pflegeleistung sagt etwas über die erforderliche Unterstützung im Lebensalltag aus. Alltagsbezogene Hilfeleistungen sind eng mit dem Wohnort assoziiert (Höpflinger 2012). Gerade die tägliche, körperbezogene Hilfe kann häufig nur im gleichen Haushalt erbracht werden. Körperbezogene Unterstützungsleistungen weisen somit im Unterschied zur organisatorischen und koordinierenden Hilfe auf eine stärkere Hilfebedürftigkeit hin.

Die Art der Gesundheitsbeeinträchtigung ist ein zusätzlicher Indikator für die Schwere der Pflegebedürftigkeit. Dabei gilt der Hilfebedarf bei kognitiver Beeinträchtigung im Vergleich zu einer rein körperlichen Behinderung oder Bewegungseinschränkung wegen der dauerhaft erforderlichen Beaufsichtigung und Anweisung bei alltäglichen Verrichtungen als höher (Schneekloth und Wahl 2005). Nicht nur bei Demenz, auch bei anderen Erkrankungen wie z. B. Multipler Sklerose erhöht sich der Pflegeaufwand, wenn (zusätzlich zur körperlichen) eine kognitive Beeinträchtigung auftritt (Buchanan et al. 2013).

Der Familienstand der erwerbstätigen Person mit Pflegeaufgaben gelangt einerseits als potenzielles Unterstützungsnetzwerk oder aber als Lebensbereich in den Blick, mit dem die Pflegetätigkeit konfligieren kann (Franke und Reichert 2011; Keck 2012). Andere Haushaltsmitglieder wie PartnerInnen und Kinder können als zusätzliche Pflegeressource die Übernahme von Pflegeaufgaben begünstigen, während Kleinkinder sie negativ beeinflussen und den zeitlichen Umfang der Pflege reduzieren.

Wie und in welcher Form ein institutionelles Versorgungsnetz die Erwerbspartizipation positiv beeinflusst, ist nicht eindeutig zu beantworten. So kommt es z. B. gerade an der Schnittstelle von privater und institutioneller Versorgung zu aufwändigen Koordinationsaufgaben (Corbin et al. 2009), die sich auf eine Vereinbarkeitssituation erschwerend auswirken können (Hoff und Hamblin 2011; Bischofberger et al. 2013). Ob professionelle Dienste die Übernahme von privaten Pflegeaufgaben begünstigen oder hemmen, können wir wegen der Anlage des Fragebogens nicht untersuchen.

\subsection{Arbeitsplatzbezogene Bedingungen}

Die beiden zentralen Merkmale einer Pflegesituation «Zeiterfordernis» und «Unplanbarkeit» bedingen ein Aufbrechen von geregelten und planbaren Arbeitszeiten, um Erwerbstätigkeit und private Pflegeaufgaben aneinander vorbei zu bringen. $\mathrm{Zu}$ den wichtigsten arbeitsplatzbezogenen Bedingungen zählen darum reduzierte und flexible Arbeitszeiten und der flexible Arbeitsort (BMFSFJ 1997; Arksey 2002; Beruf 
und Familie 2009; Hoff und Hamblin 2011). Solche Fördermassnahmen sind auch für Eltern mit (Klein-)Kindern bekannt. Für pflegende Erwerbstätige spielen Freistellungsmöglichkeiten von der Arbeitszeit eine deutlich wichtigere Rolle (BMFSFJ 2006; Colombo et al. 2011). In den meisten europäischen Ländern existieren Abwesenheitsregelungen, diese sind aber häufig entweder unbezahlt oder auf wenige Tage beschränkt (European Commission 2013; Schweizerische Eidgenossenschaft 2014). Trotz dieser Regelungen stehen diese Angebote für pflegende Erwerbstätige im Vergleich zu Eltern mit gesunden Kleinkindern weniger zur Verfügung. Neben Einkommenseinbussen könnte erschwerend hinzukommen, dass die Abwesenheit geplant werden muss, was sich gerade bei Pflegesituationen als schwierig erweist. Kümmerling und Bäcker (2011) weisen zudem darauf hin, dass die verschiedenen Massnahmen je nach Tätigkeitsfeld eines Unternehmens variieren sowie abhängig vom jeweiligen Arbeitsplatz sind, weshalb sie nicht allen Erwerbstätigen gleichermassen zur Verfügung stehen.

\section{Daten, Variablenbeschreibung und Methode}

Inwieweit die in der Literatur diskutierten Faktoren auf die Übernahme von Pflegeaufgaben durch Erwerbstätige bzw. auf die Höhe des geleisteten Pflegeumfanges einen Einfluss haben, soll mittels multivariater Regressionsmethoden untersucht werden. Datenbasis für die Untersuchung bilden zwei unterschiedliche Unternehmen, ein privatwirtschaftlicher Grosskonzern und eine mittelgrosse öffentliche Verwaltung. Beide sind im Dienstleistungssektor zu verorten, obschon in Bezug auf ihr Tätigkeitsfeld und ihre Betriebs- und Personalstruktur deutliche Unterschiede festzustellen sind. Weist das Privatunternehmen eine hohe Vollzeitquote (82\%) auf und setzt sich mehrheitlich aus männlichen Mitarbeitern (71\%) zusammen, sind etwas mehr als die Hälfte der Angestellten der Verwaltung teilzeitangestellt (57\%) und weiblich (60\%; Tabelle 1). Ferner unterscheiden sich die Unternehmen nach dem durchschnittlichen Stundenlohn. ${ }^{6}$ In Bezug auf die Verteilung des Haushaltseinkommens und des Ausbildungsniveaus ihrer Angestellten sind sich Privatunternehmen und Verwaltung aber ähnlich. ${ }^{7}$

Die Erhebung der Daten erfolgte mit Hilfe einer online durchgeführten Umfrage innerhalb der beiden Unternehmen. Beim eingesetzten Online-Fragebogen handelt es sich um ein standardisiertes, branchenunabhängiges Instrument, um betriebliche Daten zur Häufigkeit und Verteilung der Pflege- und Betreuungsarbeit der Mitarbeitenden zu erheben. Weil privat geleistete Unterstützungsaufgaben typi-

$6 \quad$ Stundenlohn nach Unternehmen: $(t(3221)=4.964, p=0.000)$. Privatunternehmen $(N=1777)$ : Mittelwert 70.0 CHF, Standardfehler 0.5, Standardabweichung 23.0, Verwaltung ( $\mathrm{N}=1446)$ : Mittelwert 66.1 CHF, Standardfehler 0.5, Standardabweichung 20.7.

7 Ausbildungsniveau nach Unternehmen: $\chi^{2}(2, N=4195)=3.164, p=0.206$, Haushaltseinkommen nach Unternehmen: $\chi^{2}(2, N=3364)=5.262, \mathrm{p}=0.072$ 
Tabelle $1 \quad$ Betriebsstruktur der beiden Unternehmen

\begin{tabular}{|c|c|c|c|c|c|c|}
\hline & \multicolumn{3}{|c|}{ Privater Dienstleistungsbetrieb } & \multicolumn{3}{|c|}{ Öffentliche Verwaltung } \\
\hline & $\begin{array}{l}\text { Grund- } \\
\text { gesamtheit }\end{array}$ & Stichprobe & $n(N)$ & $\begin{array}{l}\text { Grund- } \\
\text { gesamtheit }\end{array}$ & Stichprobe & $n(N)$ \\
\hline $\mathrm{N}$ & $>10000$ & 2436 & & $>5000$ & 1766 & \\
\hline \multicolumn{7}{|l|}{$\begin{array}{l}\text { Persönliche und } \\
\text { unternehmensspezifische } \\
\text { Merkmale }\end{array}$} \\
\hline Durchschnittsalter (Jahre) & 41 & 42 & $2427(2436)$ & 44 & 44 & $1759(1766)$ \\
\hline Frauenanteil & $29 \%$ & $26 \%$ & $641(2436)$ & $60 \%$ & $65 \%$ & $1141(1764)$ \\
\hline Teilzeitquote (1-80\%) & $18 \%$ & $13 \%$ & $324(2436)$ & $57 \%$ & $51 \%$ & $893(1766)$ \\
\hline Kader & (k.A.) & $26 \%$ & $624(2436)$ & (k.A.) & $28 \%$ & $488(1766)$ \\
\hline \multicolumn{7}{|l|}{ Bildungsniveau } \\
\hline primäres & (k.A.) & $4 \%$ & $89(2433)$ & (k.A.) & $4 \%$ & $68(1762)$ \\
\hline sekundäres & (k.A.) & $46 \%$ & $1132(2433)$ & (k.A.) & $44 \%$ & $771(1762)$ \\
\hline tertiäres & (k.A.) & $50 \%$ & $1212(2433)$ & (k.A.) & $52 \%$ & $923(1762)$ \\
\hline \multicolumn{7}{|l|}{ Haushaltseinkommen } \\
\hline tief (0-6000 CHF) & (k.A.) & $17 \%$ & $338(1893)$ & (k.A.) & $20 \%$ & $286(1471)$ \\
\hline mittel (6001-10 000 CHF) & (k.A.) & $43 \%$ & 815 (1893) & (k.A.) & $45 \%$ & $666(1471)$ \\
\hline hoch (ab 10000 CHF) & (k.A.) & $39 \%$ & 740 (1893) & (k.A.) & $35 \%$ & 519 (1471) \\
\hline $\begin{array}{l}\text { Stundenlohn (CHF pro } \\
\text { Stunde) }\end{array}$ & (k.A.) & 70 & $1777(2436)$ & (k.A.) & 66 & 1446 (1766) \\
\hline
\end{tabular}

Quelle: Eigene Daten.

scherweise in vielfältigen Formen in Erscheinung treten und nicht nur körpernahe Hilfeleistungen implizieren, wurde eine umfassende Konzeption verfolgt. ${ }^{8}$ Als erwerbstätig galten alle im Betrieb Beschäftigten, unabhängig vom Anstellungsgrad. Ebenso wenig wurde der Kreis der Pflegeempfänger eingegrenzt. ${ }^{9}$ Von Pflege mehrfach betroffene Mitarbeitende wurden gebeten, eine Person auszuwählen, auf die sie sich in der Befragung beziehen. In der Verwaltung fand eine Vollerhebung $(N>5000)$, im Privatunternehmen $(N>10000)$ eine Teilerhebung $(n=4625)$ statt. Im Privatunternehmen wurden zwei Mitarbeitergruppen nicht befragt, um Arbeitsabläufe nicht zu behindern. Da temporär Beschäftigte und Mitarbeitende mit einem Auftragsverhältnis nicht zur Kernbelegschaft des Unternehmens zählen, ist ein Ausschluss dieser Fälle insbesondere in Hinblick auf den Einbezug anstellungsspezifischer Einflussfaktoren in die Analysen sinnvoll. In den übrigen Bereichen

8 Es waren alle Hilfeleistungen gemeint, wie z. B. Körperpflege, Hausarbeiten, Administrations- und Koordinationsaufgaben, persönliche und emotionale Begleitung.

9 Bei den Pflegebedürftigen handelt es sich um Personen, die unabhängig von Verwandtschaftsgrad, Alter, Pflegedauer oder der gesundheitlichen Beeinträchtigung auf Unterstützung angewiesen sind. 


\section{Tabelle $2 \quad$ Variablenbeschreibung}

\begin{tabular}{|c|c|c|}
\hline Variable & Beschreibung & Kategorien \\
\hline \multicolumn{3}{|l|}{ Abhängige Variablen } \\
\hline Pflegeübernahme & Pflegetätigkeit, dichotom & \\
\hline Pflegeumfang & $\begin{array}{l}\text { Pflegeumfang in Stunden pro } \\
\text { Monat, logarithmiert (In) }\end{array}$ & \\
\hline \multicolumn{3}{|l|}{ Unabhängige Variablen } \\
\hline Frau & Geschlecht & $\begin{array}{l}\text { (1) Frau } \\
\text { (0) Mann }\end{array}$ \\
\hline Alter & Alter in Jahren & \\
\hline Ausbildungsniveau & $\begin{array}{l}\text { Höchster Ausbildungsabschluss, } \\
\text { Zuordnung }\end{array}$ & $\begin{array}{l}\text { Tief (kein Schulabschluss, obligatorischer } \\
\text { Schulabschluss) } \\
\text { Mittel (Lehre, Matura/Berufsmatura/ } \\
\text { Diplommittelschule, höhere Schule) } \\
\text { Hoch (Hochschule) }\end{array}$ \\
\hline Lohn pro Stellenprozent & $\begin{array}{l}\text { Persönliches Netto-Monatseinkom- } \\
\text { men geteilt durch Arbeitspensum } \\
\text { (1\%=ca. } 0.42 \text { Stunden) }\end{array}$ & \\
\hline Vollzeit & $\begin{array}{l}\text { Beschäftigungsgrad zum Zeitpunkt } \\
\text { der Pflege }\end{array}$ & $\begin{array}{l}\text { (1) Vollzeit } 81-100 \% \\
\text { (0) Teilzeit } 1-80 \%\end{array}$ \\
\hline $\begin{array}{l}\text { Anstellungspensum } \\
\text { während Pflege }\end{array}$ & $\begin{array}{l}\text { Beschäftigungsgrad während der } \\
\text { Pflegetätigkeit, metrisch }\end{array}$ & \\
\hline Leitungsfunktion & Position mit Vorgesetztenfunktion & $\begin{array}{l}\text { (1) Mit Vorgesetztenfunktion } \\
\text { (0) Ohne Vorgesetztenfunktion }\end{array}$ \\
\hline $\begin{array}{l}\text { Paarhaushalt mit } \\
\text { Kleinkind(ern) }\end{array}$ & $\begin{array}{l}\text { Paarhaushalt mit Kleinkind(ern) } \\
\text { unter } 14 \text { Jahren im gleichen } \\
\text { Haushalt }\end{array}$ & $\begin{array}{l}\text { (1) Ja } \\
\text { (0) Nein }\end{array}$ \\
\hline Mehrpersonenhaushalt & $\begin{array}{l}\text { Haushalt grösser als } 1 \text { Person, } \\
\text { in verwandtschaftlichem, per- } \\
\text { sönlichem Verhältnis (Partner, } \\
\text { Kinder über } 14 \text { Jahre oder andere } \\
\text { Personen) }\end{array}$ & $\begin{array}{l}\text { (1) Ja } \\
\text { (0) Nein }\end{array}$ \\
\hline Haushaltseinkommen & $\begin{array}{l}\text { Netto-Monatseinkommen des } \\
\text { Haushalts, Zuordnung }\end{array}$ & $\begin{array}{l}\text { Tief (unter } 6000 \text { CHF) } \\
\text { Mittel (6001-10000 CHF) } \\
\text { Hoch (über } 10000 \text { CHF) }\end{array}$ \\
\hline $\begin{array}{l}\text { im selben Haushalt/ } \\
\text { Haus }\end{array}$ & $\begin{array}{l}\text { Wohnortsnähe zur pflege- } \\
\text { bedürftigen Person }\end{array}$ & $\begin{array}{l}\text { (1) Im gleichen Haushalt oder Haus } \\
\text { (0) In anderem Haushalt oder Haus (Gehdis- } \\
\text { tanz oder weiter weg) oder stationär }\end{array}$ \\
\hline Hauptpflege & $\begin{array}{l}\text { Hauptpflegeperson oder } \\
\text { ergänzende Pflege }\end{array}$ & $\begin{array}{l}\text { (1) Hauptpflege } \\
\text { (0) Ergänzende Pflege }\end{array}$ \\
\hline
\end{tabular}


Fortsetzung der Tabelle 2.

\begin{tabular}{|c|c|c|}
\hline Variable & Beschreibung & Kategorien \\
\hline körperbezogene Hilfe & Art der Hilfeleistung & $\begin{array}{l}\text { (1) Körperbezogene Hilfe (Toilette, Essen } \\
\text { eingeben, Ankleiden, pflege-technische } \\
\text { Massnahmen) } \\
\text { (0) Nicht- körperbezogene Hilfe (Hausarbei- } \\
\text { ten, Fahrdienst, persönliche/emotionale } \\
\text { Begleitung, Organisation/Koordination, } \\
\text { Administration, Hilfe bei Versicherungs-/ } \\
\text { Rechtsfragen) }\end{array}$ \\
\hline Beeinträchtigungen & Arten der Beeinträchtigung & $\begin{array}{l}\text { (1) Körperliche Beeinträchtigung; psychische } \\
\text { Beeinträchtigung; Beeinträchtigung der } \\
\text { Denkfähigkeit; geistige Behinderung; } \\
\text { Beeinträchtigung der Mobilität } \\
\text { (0) Keine körperliche Beeinträchtigung; } \\
\text { keine psychische Beeinträchtigung; eine } \\
\text { Beeinträchtigung der Denkfähigkeit; } \\
\text { keine geistige Behinderung; keine Beein- } \\
\text { trächtigung der Mobilität }\end{array}$ \\
\hline betriebliche Angebote & $\begin{array}{l}\text { Verfügbarkeit betrieblicher Ange- } \\
\text { bote während der Pflegetätigkeit }\end{array}$ & $\begin{array}{l}\text { (1) Flexible Arbeitszeit; bezahlte Abwesen- } \\
\text { heit; unbezahlte Abwesenheit; Reduktion } \\
\text { Arbeitspensum; flexibler Arbeitsort } \\
\text { (0) Jeweiliges Angebot steht/stand nicht zur } \\
\text { Verfügung }\end{array}$ \\
\hline genutzte Angebote & $\begin{array}{l}\text { Inanspruchnahme betriebli- } \\
\text { cher Angebote während der } \\
\text { Pflegetätigkeit }\end{array}$ & $\begin{array}{l}\text { (1) Flexible Arbeitszeit oder -ort; bezahlte } \\
\text { Abwesenheit; unbezahlte Abwesenheit } \\
\text { oder Reduktion Arbeitspensum } \\
\text { (0) Keine Inanspruchnahme des jeweiligen } \\
\text { Angebotes }\end{array}$ \\
\hline
\end{tabular}

Anmerkungen: Pflegeübernahme: Fragewortlaut: «Haben Sie schon einmal vorwiegend unbezahlt Pflege- oder Betreuungsaufgaben für Familienangehörige, Freunde oder Nachbarn übernommen (z. B. Hausarbeit, Körperpflege, Fahrdienst)? a) Ja, ich pflege/betreue zurzeit, b) Ja, aber ich pflege/betreue jetzt nicht mehr, c) Nein, aber es ist sehr wahrscheinlich, dass in naher Zukunft eine Person pflegebedürftig wird, d) Nein, die Situation betrifft mich nicht.»

Pflegeumfang: Fragewortlaut: «Wie viele Stunden wenden Sie durchschnittlich pro Monat oder Woche für die Pflege / Betreuung auf?»

erfolgte die Auswahl der Probanden innerhalb bestimmter soziodemografischer bzw. anstellungsspezifischer Gruppen wie Alter, Geschlecht, Erwerbspensum und Funktionsstufe zufällig. Die Ausschöpfungsquote im Privatunternehmen betrug 54\%, bei der Verwaltung 34\%, wobei die höhere Ausschöpfungsquote im Privatunternehmen vermutlich aus der umfassenderen IT-Infrastruktur und höheren PC-Vertrautheit 
der Angestellten resultiert. ${ }^{10}$ In beiden Unternehmen sind die Teilzeitbeschäftigten leicht unterrepräsentiert. Ebenso zeigen sich Diskrepanzen zwischen Grundgesamtheit und Stichproben in Bezug auf das Geschlecht und das Durchschnittsalter. ${ }^{11}$ Es kann aber nicht von systematischen Ausfällen gesprochen werden. Weil hier weniger eine Verallgemeinerung auf die Grundgesamtheit, sondern eine multivariate Überprüfung der Aussagekraft bestimmter Einflussfaktoren angestrebt wird, werden für die Auswertungen ungewichtete Daten verwendet.

In der Tabelle 2 ist die Operationalisierung im vorherigen Kapitel skizzierten Einflussfaktoren ersichtlich.

\section{$4 \quad$ Ergebnisse}

\subsection{Pflegesituation in den beiden Unternehmen}

Im Vergleich zur Schätzung der Schweizerischen Arbeitskräfteerhebung von 11\% (BFS 2010) sowie dem im internationalen Kontext angenommenen Anteil von 10\% pflegenden Personen in der Erwerbsbevölkerung (Franke und Reichert 2011; Hoff und Hamblin 2011) zeigen sich ähnliche Ergebnisse. Beim Anteil an pflegenden Personen in der Erwerbsbevölkerung bestehen erhebliche länderspezifische Schwankungen, was mit der Altersstruktur und Erwerbsbeteiligung der Bevölkerung sowie den unterschiedlichen Erwerbsquoten von Frauen erklärt wird (European Commission 2013). Der Anteil der Befragten, welcher aktuell für eine pflegebedürftige Person sorgt, beträgt im Privatunternehmen 12\% und in der Verwaltung 15\% (Tabelle 3). Mitarbeitende mit Pflegeaufgaben sind somit in der Verwaltung häufiger zu finden als im Privatunternehmen. ${ }^{12}$ Dabei ist der höhere Anteil pflegender Mitarbeitenden nicht auf eine längere Anstellungsdauer zurückzuführen. ${ }^{13}$ Der durchschnittliche Pflegeaufwand beträgt gegen 30 Stunden pro Monat und liegt damit weit unter der als «kritisch" definierten Marke von 20 Stunden pro Woche (Schneider et al. 2001; Heitmueller 2007; Carmichael et al. 2010). Dieser Schwellenwert für einen negativen

10 Zur Berechnung der Ausschöpfungsquote wurden auch diejenigen Fälle miteinbezogen, die in den weiteren Analysen ausgeschlossen sind. Es handelt sich um Mitarbeitende, die während der Pflege nicht oder in einem anderen Unternehmen gearbeitet haben. Die Berechnung der Ausschöpfungsquote beläuft sich auf eine Stichprobengrösse von $\mathrm{N}=2523$ beim Privatunternehmen bzw. auf $\mathrm{N}=1926$ bei der Verwaltung (vgl. Tabelle 3).

11 In den Personalstatistiken der beiden Unternehmen liegen keine Angaben zu den Ausbildungsund Einkommensniveaus vor. Ferner sind die Kaderanteile der Stichprobe nicht mit denjenigen der Personalstatistik vergleichbar, weil unterschiedliche Definitionen verwendet werden.

12 Zweiseitiger exakter Test nach Fisher, $p=0.000$.

13 Anstellungsdauer in Monaten der pflegenden Mitarbeitenden: Privatunternehmen $(\mathrm{N}=567)$ : Mittelwert 218.0, Standardfehler 5.3, Standardabweichung 127.2. Verwaltung (N=523): Mittelwert 162.0, Standardfehler 5.1, Standardabweichung 116.0.

Anstellungsdauer in Monaten der Mitarbeitenden allgemein: Privatunternehmen $(\mathrm{N}=2480)$ : Mittelwert 155.5, Standardfehler 2.4, Standardabweichung 119.6. Verwaltung $(\mathrm{N}=1876)$ : Mittelwert 119.2, Standardfehler 2.4, Standardabweichung 105.6. 
Tabelle 3 Pflegeausmass, Pflegedauer und -umfang in den beiden Unternehmen

\begin{tabular}{|c|c|c|c|c|}
\hline & \multicolumn{2}{|c|}{$\begin{array}{l}\text { Privater Dienst- } \\
\text { leistungsbetrieb }\end{array}$} & \multicolumn{2}{|c|}{ Öffentliche Verwaltung } \\
\hline & $\mathrm{N}$ & $\%$ & N & $\%$ \\
\hline Anteil nichtpflegende Mitarbeitende & 1860 & 76 & 1238 & 70 \\
\hline Anteil pflegende Mitarbeitende gesamt & 576 & 24 & 528 & 30 \\
\hline davon aktuell pflegend & 297 & 12 & 271 & 15 \\
\hline davon früher pflegend, im selben Betrieb erwerbstätig & 279 & 11 & 257 & 15 \\
\hline N (in Analyse einbezogen) & 2436 & 100 & 1766 & 100 \\
\hline davon früher pflegend, in anderem Betrieb erwerbstätig & 64 & 3 & 124 & 6 \\
\hline davon früher pflegend und nicht erwerbstätig & 23 & 1 & 36 & 2 \\
\hline \multirow[t]{2}{*}{$\mathrm{N}$ (gesamt) } & 2523 & 100 & 1926 & 100 \\
\hline & N & Monate & N & Monate \\
\hline \multirow[t]{2}{*}{ Pflegedauer im Mittel } & 576 & 47.8 & 528 & 45.7 \\
\hline & N & Stunden & $\mathrm{N}$ & Stunden \\
\hline Pflegeumfang (pro Monat) im Mittel & 576 & 31.4 & 527 & 27.6 \\
\hline
\end{tabular}

Anmerkung: Weil Aussagen auf Unternehmensebene erfolgen, wurden für alle Berechnungen, wenn nicht anders ausgewiesen, nur die Fälle einbezogen, die aktuell pflegen und früher gepflegt haben und damals bereits im Privatunternehmen bzw. bei der Verwaltung tätig waren $(n=576, n=528)$. Fälle, die früher gepflegt haben und damals noch nicht im Privatunternehmen bzw. bei der Verwaltung gearbeitet haben, sind aus der Analyse ausgeschlossen ( $n=64, n=124)$. Ebenso sind Fälle, die früher gepflegt haben und damals nicht erwerbstätig waren, aus der Analyse ausgeschlossen, weil es sich nicht um eine "work \& care»-Situation handelt. In beiden Unternehmen handelt es sich bei diesen um eine Minderheit (Privatunternehmen: $n=23$, Verwaltung: $n=36$ ). Quelle: Eigene Daten.

Effekt der Pflegeaufgaben auf die Erwerbstätigkeit wurde allerdings für England auf 10 Stunden und mehr Pflege pro Woche nach unten korrigiert (King und Pickard 2013). Wie bei Bernard und Phillips (2007) weist die Variable auch in unserer Untersuchung eine erhebliche Varianz auf. ${ }^{14}$ Die durchschnittliche Pflegedauer beträgt im Privatunternehmen 4.0 Jahre und in der Verwaltung 3.8 Jahre. ${ }^{15}$ Hinsichtlich des Pflegeumfangs und der Pflegedauer divergieren die beiden Unternehmen nicht. ${ }^{16}$

14 Pflegeumfang (Stunden pro Monat): Privatunternehmen ( $\mathrm{N}=576$ ): Mittelwert 31.4, Standardfehler 2.5, Standardabweichung 59.2. Verwaltung $(\mathrm{N}=527)$ : Mittelwert 27.7, Standardfehler 1.6, Standardabweichung 37.5.

15 Pflegedauer: Privatunternehmen (Monate) $(\mathrm{N}=576)$ : Mittelwert 47.8, Standardfehler 2.7, Standardabweichung 63.9. Verwaltung $(\mathrm{N}=528)$ : Mittelwert 45.7, Standardfehler 2.6, Standardabweichung 59.0.

16 Pflegeumfang: Mann-Whitney-U $(z=-0.451, \mathrm{p}=0.652)$, Pflegedauer: Mann-Whitney-U $(z=-0.978, \mathrm{p}=0.328)$. 
Tabelle 4

Pflegespezifische Faktoren, Haushaltsformen und betriebliche Angebote

\begin{tabular}{|c|c|c|c|c|}
\hline & \multicolumn{2}{|c|}{ Privatunternehmen } & \multicolumn{2}{|c|}{ Öffentliche Verwaltung } \\
\hline & $n(N)$ & $\%$ & $n(N)$ & $\%$ \\
\hline \multicolumn{5}{|l|}{ Pflegespezifische Faktoren } \\
\hline Im selben Haushalt/Haus & $142(568)$ & 25 & $114(517)$ & 22 \\
\hline Hauptpflege & $136(576)$ & 24 & $127(528)$ & 24 \\
\hline Körperbezogene Pflege & $315(569)$ & 55 & $308(526)$ & 58 \\
\hline \multicolumn{5}{|l|}{ Beeinträchtigungen } \\
\hline körperlich & $425(576)$ & 74 & $406(528)$ & 77 \\
\hline psychisch & $53(576)$ & 9 & $84(528)$ & 16 \\
\hline Denkfähigkeit & $109(576)$ & 19 & $130(528)$ & 25 \\
\hline Mobilität & $168(576)$ & 29 & $179(528)$ & 34 \\
\hline \multicolumn{5}{|l|}{ Haushaltsformen } \\
\hline Paarhaushalt mit Kleinkind(ern) & $511(2436)$ & 21 & $335(1766)$ & 19 \\
\hline Mehrpersonenhaushalt & $1796(2436)$ & 74 & $1360(1766)$ & 77 \\
\hline \multicolumn{5}{|l|}{ Betriebliche Angebote } \\
\hline \multicolumn{5}{|l|}{ Zur Verfügung stehende Angebote } \\
\hline flexible Arbeitszeit & $2196(2436)$ & 90 & $1204(1766)$ & 68 \\
\hline flexibler Arbeitsort & $2025(2436)$ & 83 & $701(1766)$ & 40 \\
\hline bezahlte Abwesenheit & $2252(2436)$ & 92 & $1311(1766)$ & 74 \\
\hline unbezahlte Abwesenheit & $2305(2436)$ & 95 & $1465(1766)$ & 83 \\
\hline Reduktion Arbeitspensum & $2220(2436)$ & 91 & $1429(1766)$ & 81 \\
\hline \multicolumn{5}{|l|}{ Während Pflege in Anspruch genommene Angebote } \\
\hline flexible Arbeitszeiten und -orte & $327(576)$ & 57 & $193(528)$ & 37 \\
\hline Pensenreduktion oder unbezahlte Abwesenheit & $58(576)$ & 10 & $51(528)$ & 10 \\
\hline bezahlte Abwesenheit & $65(576)$ & 11 & $73(528)$ & 14 \\
\hline
\end{tabular}

Quelle: Eigene Daten.

Bei beiden Unternehmen wird am häufigsten die Pflege eines Elternteils übernommen (Privatunternehmen 59\%, Verwaltung 55\%), gefolgt von der Partnerpflege (Privatunternehmen 10\%, Verwaltung 8\%) und der Pflege eines Schwiegerelternteils (Privatunternehmen 7\%, Verwaltung 9\%). Etwas weniger häufig wird ein Grosselternteil gepflegt (je 5\%) oder Hilfe für ein pflegebedürftiges Kind übernommen (je 5\%). Pflegeaufgaben für Nachbarn und Freunde spielen nahezu keine Rolle.

Sowohl im Privatunternehmen wie auch in der Verwaltung werden die eigenen Kinder im Durchschnitt am längsten gepflegt (6.5 Jahre und länger, 7.9 Jahre und länger), Eltern oder Schwiegereltern etwa 3.5 Jahre (3.6 Jahre und länger, 3.4 Jahre und länger), Nachbarn und Freunde ca. ein Jahr (0.9 Jahre und länger, 1.3 Jahre und 
länger). Die höchste Anzahl Pflegestunden pro Monat wird im Privatunternehmen bzw. in der Verwaltung für den/die PartnerIn erbracht (54.4, 69.9), gefolgt von Stunden für ein Kind $(40.6,35.9)$ und für einen Eltern- oder Schwiegerelternteil $(23.2,23.5)$. Grosseltern, andere Verwandte und Bekannte werden im Durchschnitt zwischen 11 und 20 Stunden pro Monat gepflegt.

Tabelle 4 zeigt, dass der Anteil an körperbezogener Pflege in der Verwaltung ähnlich hoch ist wie im Privatunternehmen (55\% bzw. 58\%). Die pflegebedürftigen Personen sind am häufigsten körperlich beeinträchtigt (74\% bzw. 77\%). Etwa ein Viertel der pflegenden Befragten lebt mit der pflegebedürftigen Person im selben Haushalt oder Haus (25\% bzw. 22\%), ähnlich viele bezeichnen sich als hauptverantwortlich für die Pflege (je 24\%).

Die Möglichkeit, im Falle einer Pflegeübernahme auf die Unterstützung von Familienmitgliedern zurückzugreifen, haben in beiden Unternehmen etwa drei von vier Mitarbeitenden (Familienmehrpersonenhaushalte: 74\% bzw. 77\%). Hingegen lebt ein Fünftel der Angestellten beider Unternehmen in einer die Pflege einschränkenden Haushaltsform (Paarhaushalt mit Kleinkind[ern]: 21\% bzw. 19\%).

Den Mitarbeitenden des Privatunternehmens stehen die verschiedenen betrieblichen Angebote häufiger zur Verfügung als jenen der Verwaltung. Die Angebote wurden aber mit Ausnahme flexibler Arbeitszeiten oder -orte von den pflegenden Mitarbeitenden des Privatunternehmens nicht häufiger genutzt als von jenen der öffentlichen Verwaltung. 57\% der pflegenden Angestellten des Privatunternehmens nutzten das Angebot flexibler Arbeitszeiten oder -orte, 37\% der Mitarbeitenden der Verwaltung.

\subsection{Pflegewahrscheinlichkeit nach soziodemografischen Merkmalen, Anstellungsverhältnis und betrieblichen Angeboten}

Welche Faktoren die Übernahme von Pflegeaufgaben zu erklären vermögen, zeigen die Ergebnisse der logistischen Regression in Modell 1, Tabelle 5. Die Wahrscheinlichkeit zu pflegen liegt bei einer weiblichen Mitarbeiterin höher und steigt mit zunehmendem Alter. Die Lohnhöhe wirkt negativ. Ein Effekt des Haushaltseinkommens zeigt sich nur beim Privatunternehmen: Mitarbeitende, denen ein hohes Haushaltseinkommen zur Verfügung steht, übernehmen hier seltener Pflegeaufgaben. Die Merkmale des Anstellungsverhältnisses, der Haushaltsformen sowie das Ausbildungsniveau sind nicht relevant.

Eine Erweiterung des Modells (Modell 2, Tabelle 5) um die Variablen der betrieblichen Angebote bestätigt die im Modell 1 relevanten Faktoren Geschlecht, Alter, Stundenlohn sowie das Haushaltseinkommen beim Privatunternehmen. Bei beiden Unternehmen zeigt sich ein negativer Effekt der unbezahlten Abwesenheit auf die Übernahmewahrscheinlichkeit von Pflegeaufgaben. Die Verfügbarkeit der verschiedenen Angebote wirkt sich in den beiden Unternehmen unterschiedlich auf die Pflegeübernahme aus. Während sich in der Verwaltung die Möglichkeit 


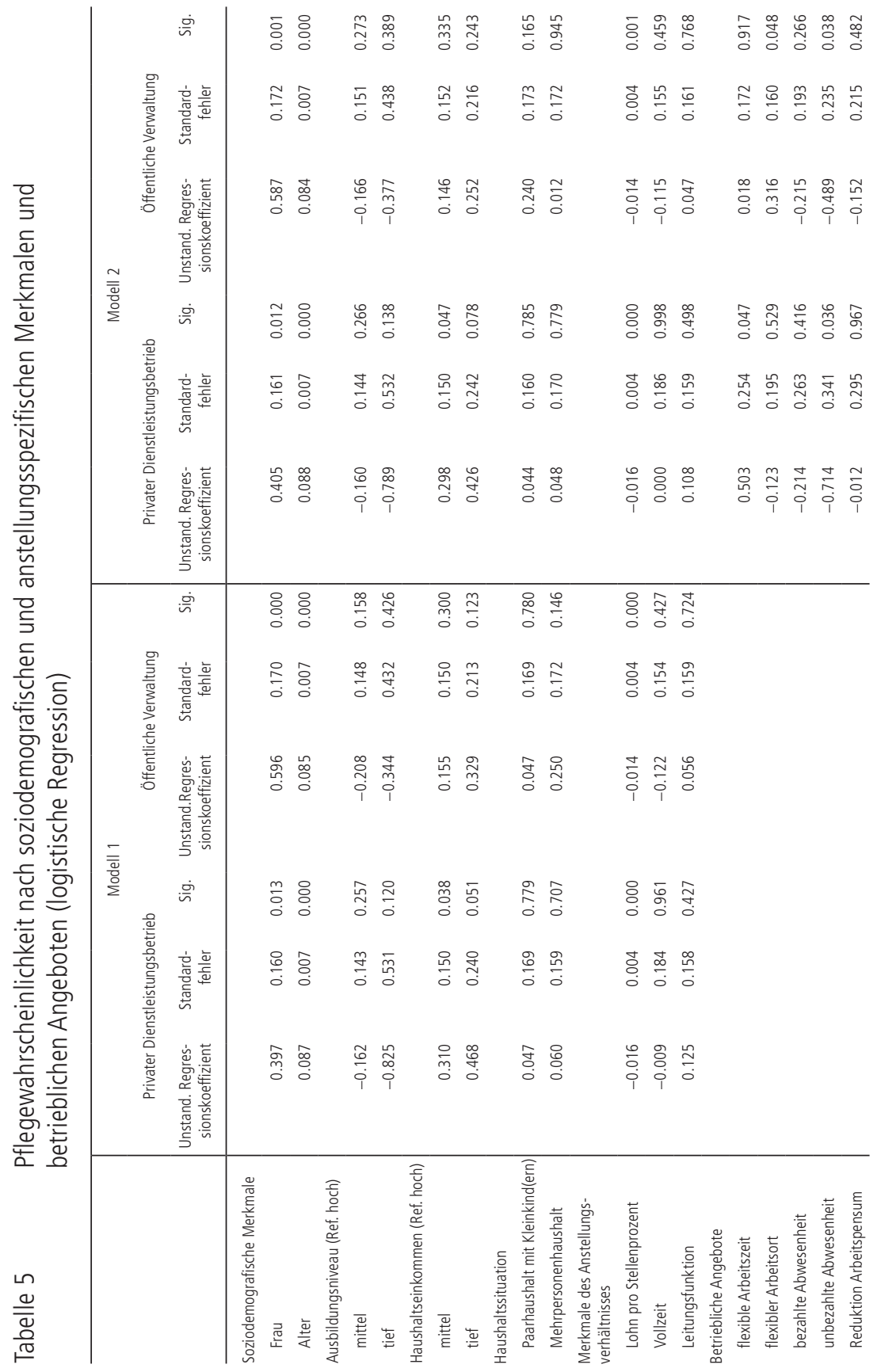




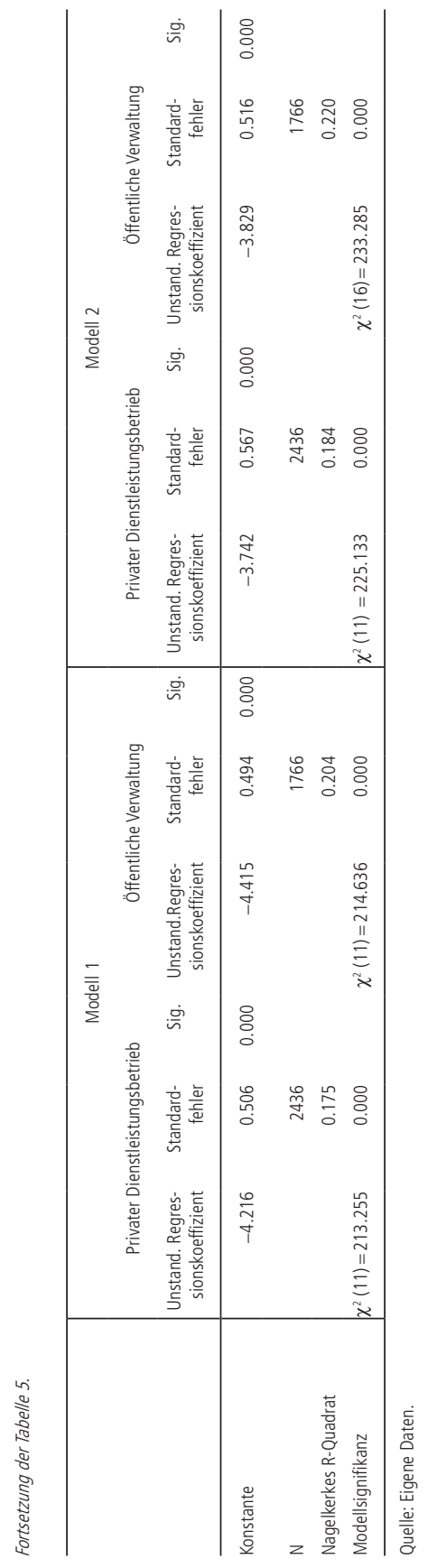


eines flexiblen Arbeitsortes positiv auf die Wahrscheinlichkeit der Pflegeübernahme auswirkt, erweist sich im Privatunternehmen das Angebot der flexiblen Arbeitszeit als aussagekräftig.

Beide Modelle weisen signifikante Modellspezifikationen auf ( $\mathrm{p} \leq 0.001)$. Ferner nimmt die Effektstärke in Modell 2 (Nagelkerkes R-Quadrat: Privatunternehmen 0.184, Verwaltung: 0.220) im Vergleich zu Modell 1 (Nagelkerkes R-Quadrat: Privatunternehmen 0.175, Verwaltung: 0.204) zu.

\subsection{Pflegeumfang nach soziodemografischen, anstellungs- und pflegespezifischen Merkmalen sowie betrieblichen Angeboten}

Die Ergebnisse der multivariaten linearen Regressionsanalyse ${ }^{17}$ zeigen, dass der Pflegeumfang als Indikator für die anfallende zeitliche Pflegebelastung der Beschäftigten praktisch ausschliesslich mit Merkmalen der Pflegesituation korreliert; die Merkmale des Anstellungsverhältnisses spielen keine Rolle (Modell 3, Tabelle 6).

Leben pflegende und pflegebedürftige Person im gleichen Haushalt, ist erwartungsgemäss der Pflegeumfang höher. Ebenso erweist sich die selbstzugeschriebene pflegerische Position «hauptpflegend» als entscheidend. Der Pflegeumfang ist höher, wenn körperbezogene Hilfe übernommen wird. Eine körperliche Beeinträchtigung der pflegebedürftigen Person erhöht allein bei der Verwaltung den Pflegeumfang. Keinen Einfluss auf die Anzahl geleisteter Pflegestunden weist die Haushaltsform auf, d. h. weder wirken sich weitere Haushaltsmitglieder positiv auf den geleisteten Pflegeaufwand aus, noch hemmen Kleinkinder in einem Familienhaushalt diesen.

Alter, Geschlecht und Haushaltseinkommen der pflegenden Person wirken sich nicht auf die geleistete Anzahl Pflegestunden aus. Allein im Privatunternehmen nimmt die Anzahl Pflegestunden mit steigendem Bildungsniveau ab: Mitarbeitende mit hohem Bildungsniveau pflegen in geringerem Umfang.

Durch die Erweiterung des Modells um die betrieblichen Angebote (Modell 4, Tabelle 6) werden weitere Unterschiede zwischen den beiden Betrieben ersichtlich. Weiterhin vermögen vor allem pflegespezifische Faktoren einen höheren Pflegeaufwand zu erklären. Beim Privatunternehmen ist jedoch der Pflegeumfang höher, wenn das Angebot der Pensenreduktion oder unbezahlten Abwesenheit in Anspruch genommen wurde, bei der Verwaltung, wenn von der Möglichkeit der bezahlten Abwesenheit Gebrauch gemacht wurde. ${ }^{18}$ Die Nutzung flexibler Arbeitszeiten oder -orte zeigt bei keinem der Unternehmen einen Effekt auf den Umfang der geleisteten Unterstützung.

17 Infolge der Verteilung der abhängigen Variable «Anzahl Stunden Pflege pro Monat» bzw. deren Residuen war eine nicht-lineare Transformation $(\ln (\mathrm{x}))$ der Variable notwendig, um diese an eine Normalverteilung anzunähern.

18 Bivariate Analysen zeigen, dass die Nutzung der Angebote und der Pflegeumfang im Zusammenhang stehen. Mitarbeitende, die Angebote nutzen, pflegen mehr (Nutzung des jeweiligen Angebotes: $\mathrm{N}>50$ ). 


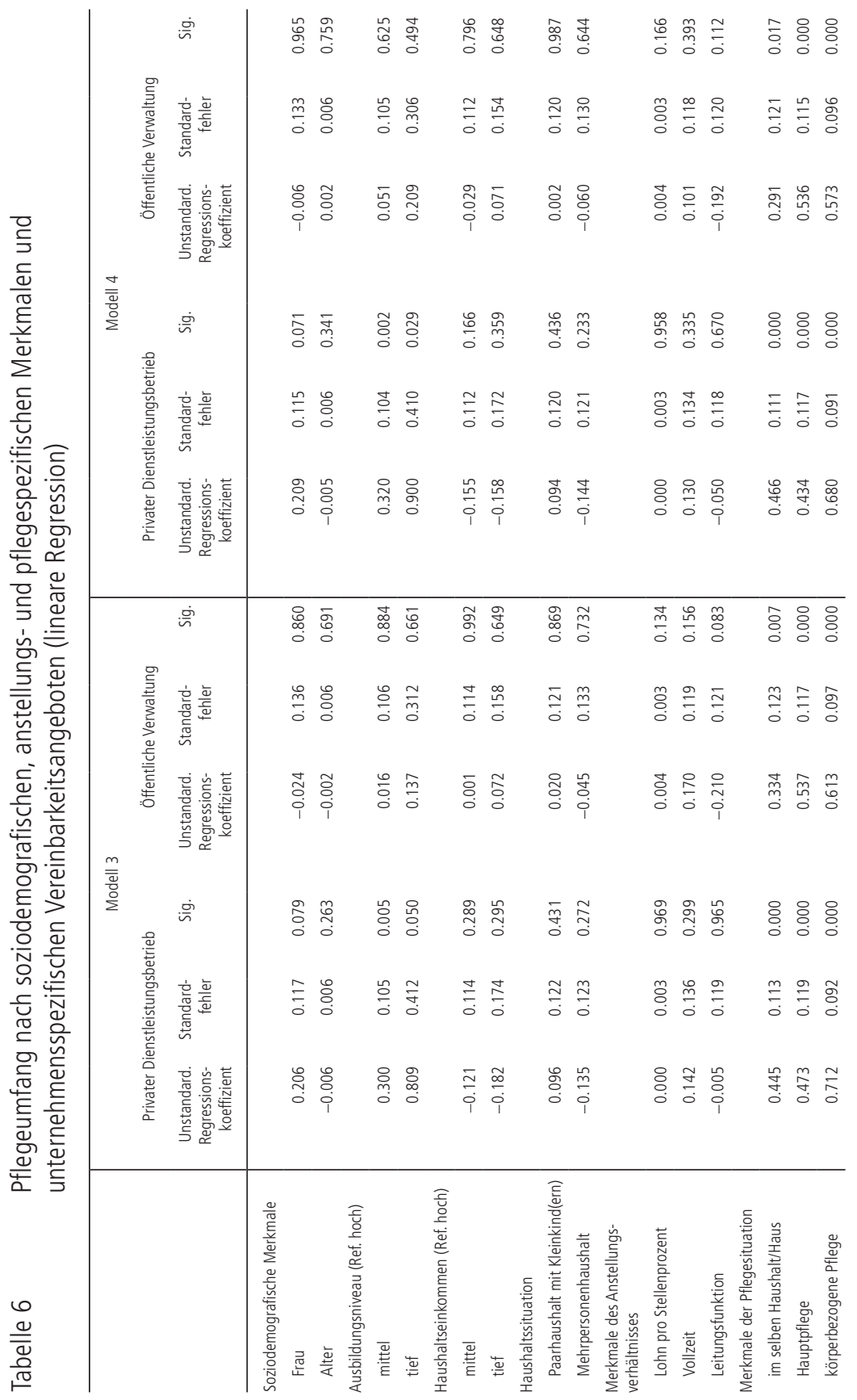




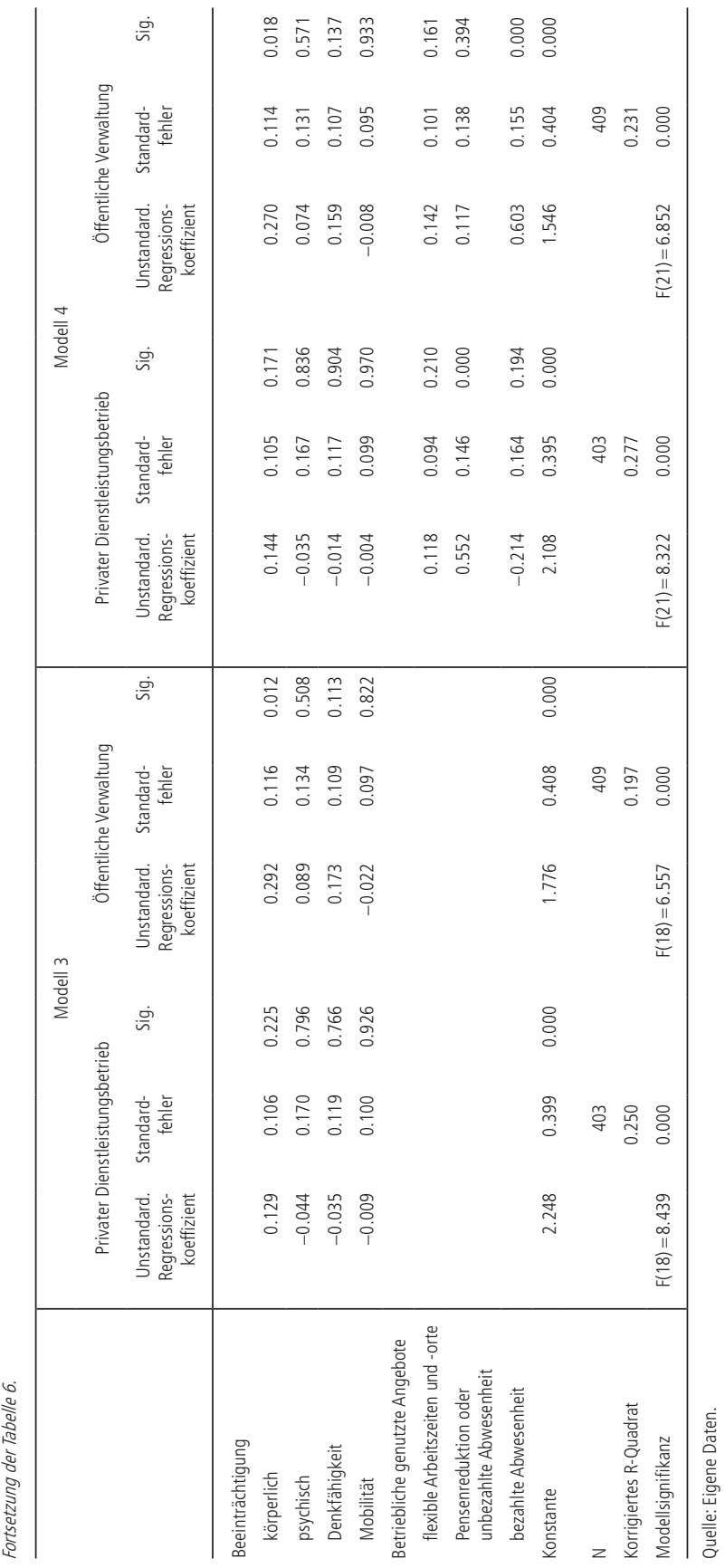


Beide Modelle sind signifikant $(\mathrm{p} \leq 0.001)$ und der Einbezug der Variablen der betrieblichen Angebote erhöht die Erklärungskraft des Modells: Der Wert des korrigierten R-Quadrats nimmt von 0.250 auf 0.277 (Privatunternehmen) bzw. von 0.197 auf 0.231 (Verwaltung) zu.

\section{Diskussion}

Die beiden Stichproben unterscheiden sich bezüglich der Pflegeübernahme, nicht aber hinsichtlich des von den Mitarbeitenden geleisteten Pflegeumfangs und der Dauer ihrer pflegerischen Unterstützung signifikant voneinander. Das Geschlecht erweist sich als signifikanter Faktor für die Pflegeübernahme in beiden Unternehmen, beim Pflegeumfang spielt es jedoch keine Rolle. Es kann folglich daraus geschlossen werden, dass Geschlechterunterschiede in der Belegschaft eines Betriebs zu unterschiedlichen Pflegehäufigkeiten führen können. Ferner ist nicht auszuschliessen, dass in bestimmten Branchen, etwa im Sozial- und Gesundheitsbereich, mit höheren Anteilen an pflegenden Mitarbeitenden zu rechnen ist (Bernard und Phillips 2007). Je älter auch die Belegschaft eines Unternehmens, umso stärker ist diese mit der Vereinbarkeitsproblematik konfrontiert, wie bereits Martin-Matthews und Rosenthal (1993) konstatieren.

Dass Frauen in beiden Unternehmen häufiger pflegen, erklärt sich damit, dass die Versorgung von Eltern und Schwiegereltern vorwiegend durch Frauen, d. h. von Töchtern und Schwiegertöchtern, übernommen wird (Hoff und Hamblin 2011). Solche Pflegekonstellationen treten in der Erwerbsphase häufiger auf als die Pflege anderer Familienmitglieder. So nimmt die Bedeutung der Partnerpflege, in die auch Männer stärker involviert sind, erst nach der Pensionierung zu (Franke und Reichert 2011).

Eine Pflegesituation tritt in beiden Unternehmen häufiger bei den älteren Erwerbstätigen auf. Ab dem 50. Lebensjahr nimmt die Pflegewahrscheinlichkeit deutlich zu. ${ }^{19}$ Dieser Befund deckt sich mit europäischen Zahlen (European Commission 2013). In der Schweiz besteht die höchste Wahrscheinlichkeit einer beruflich-pflegerischen Kombination durch die Pflegebedürftigkeit eines Elternteils im letzten Jahrzehnt vor der Pensionierung (Höpflinger 2012).

In beiden Unternehmen erweist sich der Lohn als relevant. Beim Entscheid, Pflege zu übernehmen, spielen die Opportunitätskosten der Pflege eine Rolle. Die für die Pflege aufgewendete Zeit kann als Lohn betrachtet werden, welcher der Person entgeht, wenn sie anstelle ihrer Erwerbstätigkeit Pflege übernimmt. Je höher der

19 Anteil pflegende Erwerbstätige in Privatunternehmen und Verwaltung (aktuell und früher pflegend und bereits im entsprechenden Betrieb tätig) : unter 20 Jahre: 3\%, 14\%; 20-29 Jahre: 9\%, 10\%; 30-39 Jahre: $24 \%, 13 \%$; 40-49 Jahre: 24\%, 32\%; 50-59 Jahre: 40\%, 43\%; 60 und mehr Jahre: $38 \%, 48 \%$. 
Lohn, desto höher sind die Opportunitätskosten der Pflege und umso geringer ist die Wahrscheinlichkeit, Pflege zu übernehmen.

Der von Schneider et al. (2001) begründete Zusammenhang zwischen Haushaltseinkommen und Pflegeübernahme bei erwerbstätigen verheirateten Frauen lässt sich nicht auf die allgemeine erwerbstätige Population übertragen. Einerseits zeigt sich beim Privatunternehmen ein Effekt, nicht hingegen bei der Verwaltung. Es kann vermutet werden, dass die Frage nach der finanziellen Versorgungsalternative bei der Verwaltung erst bei einer hohen Pflegeintensität zum Tragen kommt. So steht die Erwerbstätigkeit von Teilzeitmitarbeitenden erst bei einem hohen zeitlichen Unterstützungsbedarf im Konflikt mit der Pflege. Andererseits steigen nach Schneider et al. (2001) teilzeitarbeitende Frauen eher aus dem Erwerbsleben aus, wenn das Haushaltseinkommen hoch ist, weshalb sie in dieser Untersuchung nicht erfasst werden. Im Privatunternehmen weisen Mitarbeitende mit einem hohen Haushaltseinkommen eine tiefere Pflegewahrscheinlichkeit auf, als solche mit einem mittleren. Das Haushaltseinkommen scheint hier eine ökonomische Ressource zu sein, die es den Mitarbeitenden ermöglicht, Pflege auszulagern, sei es an externe Pflegedienste oder an den Partner oder die Partnerin. Zu den Mitarbeitenden mit tiefem Haushaltseinkommen zählen vor allem Junge, welche alleine wohnen. Ihre Pflegewahrscheinlichkeit unterscheidet sich deshalb nicht signifikant von denjenigen Mitarbeitern mit hohem Einkommen. ${ }^{20}$

Mit Ausnahme des Lohnes ist eine Abhängigkeit der Pflegeübernahme von den anderen anstellungsspezifischen Faktoren in keinem der Unternehmen sichtbar. Gestützt auf die Befunde von Carmichael et al. (2010) können wir die Annahme bestätigen, dass in Vollzeit erwerbstätige Personen nicht weniger häufig als in Teilzeit Beschäftigte Pflegeaufgaben übernehmen. Es ist damit nicht das Anstellungspensum einer Person, sondern ihr Geschlecht entscheidend, ob sie pflegt. Da Frauen aber vermehrt Teilzeit arbeiten, kann sich vordergründig ein Effekt des Anstellungspensums auf die Pflegeübernahme zeigen. ${ }^{21}$ Ausschlaggebend dafür, dass Frauen in beiden Betrieben häufiger Teilzeit angestellt sind, hat mit der Diskontinuität in der Erwerbstätigkeit der Frauen (Heitmueller 2007) zu tun. Ebenso spielt die Führungsverantwortung für die Pflegeübernahme keine Rolle. Personen in Kaderpositionen pflegen nicht weniger häufig. Da die Pflegewahrscheinlichkeit mit dem Alter zunimmt und Kaderpersonen im Durchschnitt älter sind, ist zu vermuten,

20 Durchschnittsalter nach Haushaltseinkommen: Verwaltung: tief: 42.5 Jahre, mittel: 44.4 Jahre, hoch: 45 Jahre; einfaktorielle ANOVA, $F(2,1465)=4.282, \mathrm{p}=0.000$. Privatunternehmen: tief: 40.3 Jahre, mittel: 43.3 Jahre, hoch: 42.2 Jahre; einfaktorielle ANOVA, $F(2,1885)=9.827, p=0.000$. Zusammenhang zwischen Einpersonenhaushalte und Haushaltseinkommen: Privatunternehmen: $\chi^{2}(2, N=1893)=230.695, p=0.000$, Verwaltung: $\chi^{2}(2, N=1471)=214.360, p=0.000$.

21 So zeigen bivariate Analysen einen signifikanten Zusammenhang zwischen der Teilzeitarbeit und dem Geschlecht in der Verwaltung (zweiseitiger exakter Test nach Fisher, $N=1766, p=0.000$ ), jedoch nicht im Privatunternehmen (zweiseitiger exakter Test nach Fisher, $N=2436, p=0.206$ ). 
dass der negative Effekt einer Kaderposition auf die Pflegewahrscheinlichkeit durch den positiven Effekt des Alters aufgehoben wird. ${ }^{22}$

Dass eine beschäftigte Person mit geringerem Ausbildungsgrad eher Pflegeaufgaben übernimmt, lässt sich für keines der Unternehmen bestätigen. Hingegen zeigt sich beim Privatunternehmen, dass mit steigendem Ausbildungsniveau der Pflegeumfang abnimmt. Im Einklang mit Schneider et al. (2001) scheinen besser Ausgebildete mehr finanzielle Versorgungsalternativen zu haben, die es ihnen ermöglichen, Pflege zumindest partiell auszulagern. Ähnlich wie beim Haushaltseinkommen spielt das Ausbildungsniveau erst dann eine Rolle, wenn ein Konfliktverhältnis zwischen Erwerbstätigkeit und Pflege auftritt. Eine Entscheidungssituation der vorwiegend Teilzeitbeschäftigten bei der Verwaltung tritt im Vergleich zu den Vollzeitbeschäftigten im Privatunternehmen erst bei einem hohen Pflegebedarf auf.

Die Familien- und Haushaltssituation zeigt weder bei der Pflegeübernahme noch beim Pflegeumfang einen Einfluss. So übernehmen weder Mitarbeitende, die zur Unterstützung auf eine weitere Person im Haushalt zurückgreifen können, eher Pflege, noch pflegen sie mehr. Auch die Annahme, dass die Anwesenheit von Kindern unter 14 Jahren die Wahrscheinlichkeit oder den Umfang der Pflege senken würde, lässt sich nicht bestätigen.

Der Pflegeumfang korreliert besonders mit der Pflegesituation. Bei beiden Unternehmen führt die Übernahme von körperbezogener Pflege zu einem höheren Pflegeaufwand. Im Verlauf der Erwerbstätigkeit wird vor allem Elternpflege erbracht, wobei nach Höpflinger et al. (2011) Töchter vor allem nicht körperbezogene Pflege übernehmen. Im Einklang mit den Ergebnissen dieser Studie zeigt sich bei beiden Betrieben kein Effekt des Geschlechts auf die Übernahme körperbezogener Pflege. ${ }^{23}$ Dieses Ergebnis steht im Kontrast zur vielfach hingewiesenen geschlechtsspezifischen Arbeitsteilung bei der Pflege, wonach Frauen vor allem körperbezogene Hilfe übernehmen (Hoff und Hamblin 2011; Franke und Reichert 2011). Hier stellt sich jedoch die Frage, ob dieser Zusammenhang auch für die erwerbstätige Population zutrifft, halten doch Franke und Reichert (2011) fest, dass Erwerbstätige im Vergleich zu nicht-erwerbstätigen Pflegenden generell weniger personenbezogene Hilfe leisten.

Der Befund, dass das Zusammenleben mit der pflegebedürftigen Person in enger räumlicher Nähe den Pflegeumfang signifikant beeinflusst, kann ebenso bestätigt werden. Wie die Pflegekonstellation im gleichen Haushalt auf den Grad der Einbindung in eine Pflegesituation hindeutet, unterscheiden sich Hauptpflegepersonen und ergänzend Pflegende in Bezug auf ihre (selbstzugeschriebene) pflegerische Verantwortung, die den Pflegeumfang mit beeinflusst. Dies gilt unabhängig vom

22 Das Durchschnittsalter in Kaderpositionen ist in beiden Unternehmen höher als dasjenige der Nichtkader: Privatunternehmen: Kader: 44.2 Jahre, Nichtkader: 41.7 Jahre $(\mathrm{t}(2425)=77.951$, $\mathrm{p}=0.000)$, Verwaltung: Kader: 47.1, Nichtkader: 43.1 Jahre $(t(1757)=76.973, p=0.000)$. waltung: zweiseitiger exakter Test nach Fisher, $N=524, p=0.058$. Privatunternehmen: zweiseitiger exakter Test nach Fisher, $\mathrm{N}=569, \mathrm{p}=0.141$. 
Geschlecht, d.h. Männer befinden sich in beiden Unternehmen nicht häufiger in ergänzender Pflegerolle. ${ }^{24}$

Die Art der Gesundheitsbeeinträchtigung der pflegebedürftigen Person wirkt sich kaum auf den Umfang der geleisteten Pflege aus. So führt allein die körperliche Beeinträchtigung und nur bei der Verwaltung zu einem höheren Pflegeumfang, dies, weil das Durchschnittsalter der pflegebedürftigen Person bei der Verwaltung höher liegt. ${ }^{25}$ Mit dem Alter einer Person nehmen die Einschränkung in der Beweglichkeit und die körperliche Beeinträchtigung infolge von Erkrankungen des Bewegungsapparats zu (Schneekloth und Wahl 2005). Was die kognitive Beeinträchtigung bei Demenz betrifft, nimmt mit zunehmendem Schweregrad der Krankheit, d. h. höherem Pflegeaufwand der Anteil der erwerbstätigen Pflegepersonen ab (Schneekloth und Wahl 2005). Auch Buchanan et al. (2013) weisen nach, dass eine kognitive Beeinträchtigung der an Multipler Sklerose erkrankten Person mit einer reduzierten Erwerbstätigkeit der hilfeleistenden Person assoziiert ist. Deswegen ist zu vermuten, dass Personen, die in die Pflege von kognitiv Beeinträchtigten mit einem hohen Pflegeumfang involviert sind, ihre Erwerbstätigkeit aufgeben oder zumindest unterbrechen, und somit in den hier verwendeten Stichproben nicht einbezogen sind.

Bei den unternehmensspezifischen Merkmalen zeigen sich folgende Effekte auf die Pflegeübernahme: Im Privatunternehmen, in welchem Teilzeitarbeit wenig verbreitet ist, begünstigt das verfügbare betriebliche Angebot an flexiblen Arbeitszeiten die Pflegeübernahme. Bei der Verwaltung mit einer ausgeprägten Teilzeitkultur sind dagegen Gelegenheiten wichtig, den Arbeitsort flexibel zu gestalten. Beide Befunde verweisen darauf, dass Erwerbstätigkeit und Pflegeaufgaben zeitlich aufeinander abgestimmt werden müssen. Die Flexibilität von Arbeitszeit und -ort bietet den Mitarbeitenden die Möglichkeit, die Arbeit für die Pflege unterbrechen zu können bzw. beiden Verpflichtungen nachzukommen (Hoff und Hamblin 2011). Das Angebot der unbezahlten Abwesenheit wirkt sich dagegen bei beiden Unternehmen negativ auf die Pflegeübernahme aus. Dieses Angebot mag ein Instrument für Notfälle sein, für die Pflegesituation, die eine kontinuierliche Verfügbarkeit über eine längere Zeitdauer erfordert, erweist es sich aber als ungeeignet. Ausserdem kommt es zu Einkommensverlusten, die pflegende Mitarbeitende zusätzlich zu Pflegekosten in Kauf nehmen müssten (Colombo et al. 2011).

Zwischen den Unternehmen wird ein weiterer Unterschied deutlich. Im Privatunternehmen ist der Pflegeumfang höher, wenn das Pensum reduziert oder unbezahlt Abwesenheit in Anspruch genommen wurde. Folglich reduziert sich in einem Betrieb mit hohem Anteil an Vollzeit-Beschäftigung bei höherem Pflegeaufwand das Spektrum an Vereinbarkeitsmassnahmen, die nicht mit Einkommenseinbussen

24 Bivariate Analysen des Zusammenhangs zwischen Geschlecht und Pflegerolle: Verwaltung: zweiseitiger exakter Test nach Fisher, $N=526, p=0.252$. Privatunternehmen: zweiseitiger exakter Test nach Fisher, $\mathrm{N}=576, \mathrm{p}=0.594$.

25 Durchschnittsalter der pflegebedürftigen Person: Verwaltung 72.1 Jahre $(\mathrm{N}=528)$, Privatunternehmen 68.5 Jahre $(\mathrm{N}=576)(\mathrm{t}(1102)=-2.920, \mathrm{p}=0.004)$. 
verbunden sind. Anders bei der Verwaltung, bei der das zeitliche Konkurrenzverhältnis von Erwerbstätigkeit und Pflege durch die Teilzeitbeschäftigung entschärft ist. Hier bleibt zu fragen, inwiefern die im Bundesratsbericht aufgeführte Massnahme, einen Betreuungsurlaub mit oder ohne Lohnfortzahlung zu prüfen, greifen kann (Schweizerische Eidgenossenschaft 2014). Dass bei höherem Pflegeaufwand Flexibilitätsangebote weder bei der Verwaltung, noch im Privatunternehmen greifen, verweist auf die Limitation solcher Massnahmen. Flexible Arbeitszeiten und -orte lösen den Zeitdruck nicht, zumal die Arbeit nachgeholt werden muss (Hoff und Hamblin 2011). Bei einem höheren Pflegeaufwand rückt die zeitliche Koordination von Pflege und Erwerbstätigkeit zu Gunsten der zeitlichen Verfügbarkeit per se in den Hintergrund.

\section{Konklusion}

Bei der Übernahme von privaten Pflegeaufgaben geht es um die Verteilung der Ressourcen Zeit und Geld. Anhand der beiden Unternehmensstichproben und der in die Modelle einbezogenen Variablen wurde an verschiedenen Stellen deutlich, dass Pflege einerseits Zeit erfordert und mit der Erwerbstätigkeit ausbalanciert werden muss, andererseits ökonomische Abwägungen bei der Pflegeübernahme eine Rolle spielen. Einerseits ergeben sich durch die Pflege Auswirkungen auf die Erwerbstätigkeit, wodurch Einkommenseinbussen und damit indirekt Kosten in Kauf zu nehmen sind. Andererseits können die betrieblichen Angebote eines Unternehmens diesen Balanceakt zwischen Erwerbstätigkeit und Pflege erleichtern, indem mittels flexiblen Arbeitszeiten und -orten Instrumente zur Verfügung stehen, um die Arbeit auch unvorhergesehen unterbrechen zu können. Grenzen für die Vereinbarkeit sind beispielsweise dann gesetzt, wenn Vollzeitbeschäftigung die Norm darstellt oder Präsenzzeiten erforderlich sind. Aus betrieblicher Sicht ist darum neben flexiblen Arbeitsgestaltungsoptionen vor allem auch eine Systematisierung der unerwarteten oder geplanten Abwesenheit notwendig, um kurz- und längerfristige Abwesenheiten von Mitarbeitenden zu organisieren.

Das Spannungsverhältnis zwischen Erwerbstätigkeit und Pflege ist nicht nur, wie in unserer Studie angenommen, als ein unmittelbarer zeitlicher und finanzieller Konflikt zwischen den beiden Lebensbereichen zu verstehen. Die Auswirkungen einer Pflegesituation auf die Erwerbstätigkeit können sich auch zu einem späteren Zeitpunkt manifestieren, sowohl wenn sich die Pflegedauer über mehrere Jahre erstreckt als auch nach einer veränderten oder beendeten Versorgungssituation (z. B. Heimeintritt, Tod). Bedingt durch die Pflege als eine spezifische Lebenserfahrung kann sich die Bedeutung der Erwerbstätigkeit verschieben. Dies führt zu einer Veränderung des Erwerbspartizipations- oder Berufsmusters, beispielsweise durch Unterbrüche, berufliche Umorientierung oder frühzeitiges Ausscheiden aus der 
Berufstätigkeit (Bischofberger et al. 2013). Es konkurrieren nicht nur Pflege und Erwerbstätigkeit. Sie konkurrieren auch mit anderen Lebensbereichen wie Freizeit, soziale Kontakte, Familie oder eigene Gesundheit. Ein «neuer» Zeitkonflikt kann sich nach einer Pflegesituation zeigen, wenn die einem während der Pflege entgangene Freizeit oder verpflichtungsfreie Zeit durch einen Unterbruch oder eine Reduktion der Erwerbstätigkeit nachträglich kompensiert wird. Damit sind die entscheidenden Limiten von Querschnittuntersuchungen angesprochen: Um Modifikationen der Erwerbstätigkeit im Zeitverlauf zu erfassen, wären Längsschnittuntersuchungen notwendig, die diese Prozessperspektive berücksichtigen.

Die beiden Unternehmensstichproben sind nicht bevölkerungsrepräsentativ, berücksichtigt wurden allein Erwerbstätige. Damit ist eine weitere Einschränkung mit dieser Studie verbunden. Selbstständige sind ebenso nicht erfasst wie pflegende Angehörige, die nicht ins Erwerbsleben einsteigen oder aus dem Erwerbsleben ausgestiegen sind. Ferner dominieren bestimmte Verwandtschaftskonstellationen, wie die Eltern- und Schwiegerelternpflege. Beim Verwandtschaftsverhältnis besteht aber, wie anhand der beiden referierten Studien von Heitmueller (2007) und Schneider et al. (2001) aufgezeigt wurde, weiterer Erkenntnisbedarf. Nicht nur werden unterschiedliche Arten der Pflege erbracht, die mit entsprechend unterschiedlichem Pflegeumfang verbunden sind. Es ist auch davon auszugehen, dass je nach Beziehungskonstellation die Verpflichtungsmotive divergieren (Perrig-Chiello 2012a), die den Entscheidungsspielraum bei der Übernahme von Pflegeaufgaben und den Pflegeumfang mitbeeinflussen können. Nicht zuletzt unterscheidet sich auch die Dauer der Pflegeverantwortung, was Implikationen auf die Erwerbstätigkeit haben kann. Schliesslich beeinflussen auch die Angebote des Versorgungssystems und deren Ausgestaltung den Entscheidungsspielraum der Angehörigen, ob sie Aufgaben delegieren und so den Pflegeaufwand zugunsten der Erwerbstätigkeit reduzieren können (European Commission 2013; Bischofberger und van Holten 2015).

\section{$7 \quad$ Literaturverzeichnis}

Arksey, Hilary. 2002. Combining informal care and work: Supporting carers in the workplace. Health and Social Care in the Community 10(3): 151-161.

Barkholdt, Corinna und Vera Lasch. 2006. Vereinbarkeit von Pflege und Erwerbstätigkeit. S. 261-361 in Förderung der Beschäftigung älterer Arbeitnehmer: Voraussetzungen und Möglichkeiten. Expertisen zum Fünften Altenbericht der Bundesregierung, hrsg. von Deutsches Zentrum für Altersfragen. Münster: Lit.

Baumbusch, Jennifer und Alison Phinney. 2014. Invisible hands: The role of highly involved families in long-term residential care. Journal of Family Nursing 20(1): 73-97.

Bernard, Miriam und Judith E. Phillips. 2007. Working carers of older adults: What helps and what hinders in juggling work and care? Community, Work and Family 10(2): 139-160. 
Beruf und Familie. 2009. Eltern pflegen: so können Arbeitgeber Beschäftigte mit zu pflegenden Angehörigen unterstützen - Vorteile einer familienbewussten Personalpolitik. Frankfurt a. M.: Beruf und Familie gGmbH - eine Intiative der Gemeinnützigen Hertie-Stiftung.

BFS (Bundesamt für Statistik). 2010. Schweizerische Arbeitskräfteerhebung SAKE Unbezahlte Arbeit (Spezialauswertung). Neuenburg: BFS.

BFS (Bundesamt für Statistik). 2011. Geschätzte Anzahl der Menschen mit Behinderungen. Neuenburg: BFS, http://www.bfs.admin.ch/bfs/portal/de/index/themen/20/06/blank/key/01.html (6.5.2014).

BFS (Bundesamt für Statistik). 2012. SOMED-Statistik. Neuenburg: BFS, http://www.bfs.admin.ch/ bfs/portal/de/index/themen/14/04/02/key/01.html (5.5.2014).

BFS (Bundesamt für Statistik). 2013a. Zukünftige Bevölkerungsentwicklung. Neuenburg: BFS, http:// www.bfs.admin.ch/bfs/portal/de/index/themen/01/03/blank/key/ind_erw.html (10.3.2014).

BFS (Bundesamt für Statistik). 2013b. Lebenserwartung bei guter Gesundheit nach Geschlecht, bei Geburt und im Alter von 65 Jahren. Daten. Neuenburg: BFS, http://www.bfs.admin.ch/bfs/ portal/de/index/themen/13/04/02/data/01.html (10.3.2014).

Bischofberger, Iren. 2012. Erwerbstätige pflegende Angehörige - drei zentrale Handlungsfelder. Soziale Sicherheit CHSS (1): 6-8.

Bischofberger, Iren, Andrea Radvanszky, Karin van Holten und Anke Jähnke. 2013. Berufstätigkeit und Angehörigenpflege vereinbaren. S. 162-184 in Who cares? Pflege und Solidarität in der alternden Gesellschaft, hrsg. von Schweizerisches Rotes Kreuz. Zürich: Seismo Verlag.

Bischofberger, Iren und Karin van Holten. 2015. Berufstätige Angehörige im Gesundheitswesen: Von der Versorgungslogik zur Vereinbarkeitslogik. International Journal of Helath Professions 2(1): 38-48.

BMFSFJ (Bundesministerium für Familie, Senioren, Frauen und Jugend). 1997. Betriebliche Massnahmen zur Unterstützung pflegender Arbeitnehmerinnen und Arbeitnehmer. Stuttgart: Kohlhammer.

BMFSFJ (Bundesministerium für Familie, Senioren, Frauen und Jugend). 2006. Erwartungen an einen familienfreundlichen Betrieb. Berlin: BMFSFJ, http://www.bmfsf.de/RedaktionBMFSFJ/Abteilung2/Pdf-Anlagen/erwartungen-an-einen-familienfreundlichen-betrieb, property=pdf,bereich=b mfsfi,sprache=de, $r w b=$ true.pdf (5.5.2014).

Buchanan, Robert J., Chunfeng Huang und Zhida Zheng. 2013. Factors affecting employment among informal caregivers assisting people with Multiple Sclerosis. International Journal of MS Care 15(4): 203-210.

Carmichael, Fiona und Susan Charles. 1998. The labour market costs of community care. Journal of Health Economics 17(6): 747-765.

Carmichael, Fiona, Susan Charles und Claire Hulme. 2010. Who will care? Employment participation and willingness to supply informal care. Journal of Health Economics 29(1): 182-190.

Colombo, Francesca, Ana Llena-Nozal, Jérôme Mercier und Frits Tjadens. 2011. Help Wanted? Providing and Paying for Long-term Care. OECD Health Policy Studies. OECD Publishing, http://dx.doi. org/10.1787/9789264097759-en (8.2.2016).

Corbin, Juliet, Bruno Hildenbrand und Doris Schaeffer. 2009. Das Trajektkonzept. S. 55-74 in Bewältigung chronischer Krankheit im Lebenslauf, hrsg. von Doris Schaeffer. Bern: Huber.

Creedon, Michael. 1995. Eldercare and work research in the United States. S. 93-115 in Working Carers: International Perspectives on Working and Caring for Older People, hrsg. von Judith Phillips. Aldershot: Avebury.

Dallinger, Ursula. 1997. Ökonomie der Moral. Konflikt zwischen familiärer Pflege und Beruf aus handlungstheoretischer Perspektive. Opladen: Westdeutscher Verlag.

Department of Health. 2014. Care and support statutory guidance. Issued under the Care Act 2014. London: Crown copyright, www.gov.uk/government/publications (6.3.2015). 
Destatis (Statistisches Bundesamt). 2013. Datenreport 2013. Ein Sozialbericht für die Bundesrepublik Deutschland. https://www.destatis.de/DE/Publikationen/Datenreport/Downloads/Datenreport2013.pdf?_blob=publicationFile (9.2.2016).

Deutscher Bundestag. 2014. Gesetz zur besseren Vereinbarkeit von Beruf und Pflege vom 23. Dezember 2014. Bundesgesetzblatt Jahrgang 2014 Teil 1, Nr. 64. Bonn: Bundesgesetzblatt, http://www.bgbl. de/banzxaver/bgbl/start.xav?start=\%2F\%2F*[\%40attr_id\%3D\%27bgbl114s2462.pdf\%27]\# bgbl_\%2F\%2F*[\%40attr_id\%3D\%27bgbl114s2462.pdf\%27]_1426119480084 (10.1.2015).

European Commission. 2013. The indirect costs of long-term care. Research Note 8. http://www.euro. centre.org/data/1417699093_57222.pdf (9.2.2016).

Franke, Annette und Monika Reichert. 2011. Analyse der internationalen Forschungsliteratur. Carers@ Work: zwischen Beruf und Pflege - Konflikt oder Chance? Ein europäischer Vergleich. Dortmund: Technische Universität, http://www.carersatwork.tu-dortmund.de/download/Literature\%20 review.pdf (10.3.2014).

Heitmueller, Axel. 2007. The chicken or the egg? Endogeneity in labor market participation of informal carers in England. Journal of Health Economics 26(3): 536-559.

Hoff, Andreas und Kate Hamblin. 2011. International Report. Carers@Work: Carers Between Work and Care - Conflict or Chance? Oxford: University of Oxford, Institute of Population Ageing, http:// www.carersatwork.tu-dortmund.de/download/VW\%20CarersAtWork\%20Comparative $\% 20$ Report.pdf (10.3.2014).

Höpflinger, François. 2012. Altern in der Schweiz: demografische, gesundheitliche und gesellschaftliche Entwicklungen. S. 29-62 in Pflegende Angehörige älterer Menschen: Probleme, Bedürfnisse, Ressourcen und Zusammenarbeit mit der ambulanten Pflege, hrsg. von Pasqualina Perrig-Chiello und François Höpflinger. Bern: Huber.

Höpflinger, François, Lucy Bayer-Oglesby und Andrea Zumbrunn. 2011. Informelle Hilfe und Pflege: familiale und ausserfamiliale Unterstützungsnetzwerke. S. 67-84 in Pflegebedürftigkeit und Langzeitpflege im Alter. Aktualisierte Szenarien für die Schweiz. Bern: Huber.

Keck, Wolfgang. 2012. Die Vereinbarkeit von häuslicher Pflege und Beruf. Bern: Huber.

King, Derek und Linda Pickard. 2013. When is a carer's employment at risk? Longitudinal analysis of unpaid care and employment in midlife in England. Health and Social Care in the Community 21(3): 303-314.

Kümmerling, Angelika und Gerhard Bäcker. 2011. Betriebliche Massnahmen zur Verbesserung der Vereinbarkeit von Erwerbstätigkeit und Pflegeverpflichtung. Carers@Work: zwischen Berufund Pflege - Konflikt oder Chance? Ein europäischer Vergleich. Duisburg: Universität Duisburg-Essen, http:// www.carersatwork.tu-dortmund.de/download/CarersAtWork_final_June_2012.pdf (3.5.2014).

Martin-Matthews, Anne und Judith Phillips. 2008. Aging and Caring at the Intersection of Work and Home Life. Blurring the boundaries. London: Lawrence Erlbaum Associates.

Martin-Matthews, Anne und Carolyn J. Rosenthal. 1993. Balancing work and family in an aging society: The Canadian experience. Annual Review of Gerontology and Geriatrics 13: 96-119.

Mnich, Eva, Cristian Balducci, Barbro Krevers, Kevin McKee, Costis Prouskas und Beata Wojszel. 2008. Typology of caregiving situations in Europe: A cluster analysis approach. S. 145-163 in Supporting Family Carers of Older People in Europe - The trans-European survey report, hrsg. von Giovanni Lamura, Hanneli Döhner und Christopher Kofahl. Hamburg: Eurofamcare.

NAC AARP (National Alliance for Caregiving in collaboration with AARP). 2009. Caregiving in the U.S. Bethesda und Washington: NAC und AARP, http://www.caregiving.org/data/Caregiving_in_the_US_2009_full_report.pdf (10.3.2014).

Perrig-Chiello, Pasqualina. 2012a. Familiale Pflege: ein näherer Blick auf eine komplexe Realität. S. 111-210 in Pflegende Angehörige älterer Menschen: Probleme, Bedürfnisse, Ressourcen und Zusammenarbeit mit der ambulanten Pflege, hrsg. von Pasqualina Perrig-Chiello und François Höpflinger. Bern: Huber. 
Perrig-Chiello, Pasqualina. 2012b. Einleitung. S. 17-25 in Pflegende Angehörige älterer Menschen: Probleme, Bedürfnisse, Ressourcen und Zusammenarbeit mit der ambulanten Pflege, hrsg. von Pasqualina Perrig-Chiello und François Höpflinger. Bern: Huber.

Scharlach, Andrew E. 1994. Caregiving and employment: Competing or complementary roles? Gerontologist 34(3): 378-385.

Schneekloth, Ulrich und Hans Werner Wahl (Hrsg). 2005. Möglichkeiten und Grenzen selbständiger Lebensführung in privaten Haushalten (MuG III): Repräsentativbefunde und Vertiefungsstudien $z u$ häuslichen Pflegearrangements, Demenz und professionellen Versorgungsangeboten: integrierter Abschlussbericht im Auftrag des BMFSFJ. München, http://www.bmfsfj.de/doku/Publikationen/ mug/01-Redaktion/PDF-Anlagen/gesamtdokument,property=pdf,bereich=mug,sprache=de,rw $\mathrm{b}=$ true.pdf $(6.5 .2014)$.

Schneider, Thorsten, Sonja Drobnič und Hans-Peter Blossfeld. 2001. Pflegebedürftige Personen im Haushalt und das Erwerbsverhalten verheirateter Frauen. Zeitschrift für Soziologie 30(5): 362-383.

Schweizerische Eidgenossenschaft. 2014. Unterstützung für betreuende und pflegende Angehörige. Situationsanalyse und Handlungsbedarf für die Schweiz. Bericht des Bundesrates vom 5. Dezember 2014. Bern, http://www.bag.admin.ch/themen/gesundheitspolitik/14437/index.html?lang=de (6.3.2015). 